\title{
Performance of a Crossed Exponentially Tapered Slot Antenna for UWB Systems
}

\author{
Jorge R. Costa, Member, IEEE, Carla R. Medeiros, and Carlos A. Fernandes, Senior Member, IEEE
}

\begin{abstract}
A compact printed antenna is described that exhibits adequate transient performance for ultrawideband (UWB) applications and it is further adequate for polarization diversity schemes. The antenna is based on an original combination of two crossed exponentially tapered slots plus a star-shaped slot to produce a stable radiation pattern with very stable polarization over the 3.1-10.6 GHz FCC assigned band. Results are confirmed with measurements. Figures of merit like output pulse fidelity and time window containing $90 \%$ of the transmitted energy are analyzed over the entire solid angle and showed to remain quite stable, in line with envisaged UWB system requirements. Compact dual-antenna arrangements are also analyzed in view of potential use for UWB multiple-input-multiple-output implementations.
\end{abstract}

Index Terms-Antenna transient analysis, linear polarization, printed broadband antenna, tapered slot antenna, ultrawideband (UWB) antennas.

\section{INTRODUCTION}

$\mathbf{U}$ LTRAWIDEBAND (UWB) technology has been receiving increased interest as a promising solution for extremely high data rate short-range wireless indoor applications. The operation mode of these radio systems is based on transmission/reception of trains of very short pulses, which require a large bandwidth. In the United States, the Federal Communications Commission FCC [1] authorized the unlicensed use of the $3.1-10.6 \mathrm{GHz}$ spectrum for UWB applications with limited power spectral density emission to enable spectrum sharing with other established narrowband applications without mutual interference.

Antennas play a critical role in the UWB radio performance, since they influence the shape of the output pulse, acting as a band-pass filter both in frequency and in spatial domains. So, further to the classical frequency domain analysis of antenna impedance match, polarization, gain and radiation pattern over the UWB band, also the antenna pulse-preserving capability must be appropriately considered in the antenna design. The latter can be evaluated through two figures of merit: the fidelity factor [2] and the pulse stretch ratio [3].

Manuscript received August 25, 2008; revised December 05, 2008

J. R. Costa is with Instituto de Telecomunicações, IST, 1049-001 Lisboa, Portugal and also with the Instituto Superior de Ciências do Trabalho e da Empresa, Departamento de Ciências e Tecnologias da Informação, 1649-026 Lisboa, Portugal (e-mail: Jorge.Costa@1x.it.pt).

C. R. Medeiros and C. A. Fernandes are with the Instituto de Telecomunicações, IST, 1049-001 Lisboa, Portugal.

Color versions of one or more of the figures in this paper are available online at http://ieeexplore.ieee.org.

Digital Object Identifier 10.1109/TAP.2009.2016727
Plenty of antenna solutions are proposed in the literature, seeking for the best compromise between performance and desirable compactness, low-profile and low-cost objectives. Printed antennas are the most popular choice to come near the above requirements, with especial interest for those configurations where the radiating element and the ground plane are co-planar or at least are printed on each side of a single dielectric substrate [4]. Most of these planar UWB antenna solutions can be catalogued into two broad groups: dipole/monopole-like and slot-based antennas.

Examples of wideband planar dipole type antennas include bow tie, diamond, elliptical and circular antenna [5]-[8]. But a larger number of solutions are based on planar monopole antennas [3], [9]-[11]. These monopoles and dipoles can provide extremely wide bandwidth with ratios of up to 1:20. However, the radiation patterns tend to present strong variation with frequency, in most cases with high level of cross-polarization and shifting phase centre position [11]. This has negative impact on pulse shape stability over the solid angle, which is a most important requirement for UWB applications.

Planar slot-based antennas are a competing approach for UWB applications [12], [13]. Although, some designs also tend to present radiation pattern stability and cross-polarization problems, tapered slot configurations [14], [15] have the advantage of usually producing a smooth bidirectional patch-like radiation pattern with low cross-polarization.

Many other non-planar solutions are also reported, including vertical monopoles with ground plane [16], [17], horn-based antennas [18], [19] and volume dielectric antennas [20], [21] for increased directivity. Some of these solutions bare the same radiation stability issues versus frequency in the solid angle of observation, but bulk is the main drawback.

A large number of reported UWB antennas are designed and optimized without paying attention to the transient response. Also polarization stability is generally disregarded considering it of little relevance, especially for personal terminal communications. However, diversity schemes are being considered to enhance UWB radio performance [22], [23]; so issues like mutual coupling in multi-antenna systems and polarization purity become relevant in this case.

The present paper proposes and evaluates the feasibility of a new UWB antenna complying with the above requirements. It is a printed planar slot-based antenna, with double-symmetry and balanced configuration. The operation bandwidth covers the whole 3.1 to $10.6 \mathrm{GHz}$ UWB band. The antenna is very compact $\left(0.3 \lambda_{0} \times 0.3 \lambda_{0}\right)$, low profile, with very pure stable linear polarization, low pulse distortion and negligible coupling to adjacent antennas in multi-antenna assemblies. The proposed balanced 

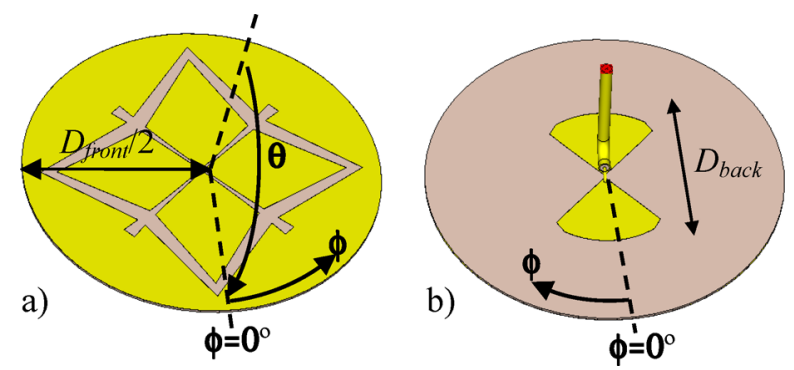

Fig. 1. XETS antenna geometry in CST Microwave Studio [28] simulation model: a) front face; b) back face.

antenna configuration is adequate for direct integration of differential RFICs at its back, without RF cables or additional balun [24]. UWB chip transceivers design based on differential signal paths are becoming standard practice [25], [26] so the balanced antenna configuration is the preferred choice in this case.

A detailed study of the antenna frequency domain characteristics and impulse response is carried out in this paper. Simulated and measured results are presented which confirm the potential of the proposed antenna for UWB systems. A feasibility study of the proposed antenna configuration, based just on simulations, was presented in [27] for a different 1:3 frequency interval.

This paper is organized as follows. The antenna concept is presented in Section II. The antenna performance is presented in Sections III for the frequency domain and in Section IV for time domain. Mutual coupling in multi-antenna arrangements is addressed in Section V. Numerical and experimental results are given and discussed throughout. Conclusions are finally drawn in Section VI.

\section{ANTENNA DESCRIPTION}

The antenna geometry is presented in Fig. 1. The front face is composed of a circular metal layer with two crossed exponentially tapered slots, intersected by a star like slot. The metal layer circumference has diameter $D_{\text {front }}$. Chopped replicas of two opposing "petals" of the antenna are printed at the back-side of the substrate, Fig. 1(b). The chopped edge of these petals is an arc of circumference with diameter $D_{\text {back }}$. The antenna is fed between the two back petals, which couple capacitively at RF with the corresponding front petals of the antenna. The $\phi=0$ plane as shown in Fig. 1 defines the antenna E-plane. It passes through the petals that are fed by the coaxial cable. The $\phi=90^{\circ}$ plane defines the antenna H-plane.

The antenna perfect symmetry with respect to feed point ensures perfect symmetry of the antenna currents with respect to the E-plane and perfect anti-symmetry with respect to the H-plane, irrespective to frequency. Consequently, pure linear polarization versus frequency is obtained at least in the main planes. The slots in the front face metallization determine the radiation pattern, which is bidirectional due to the absence of a ground plane. A single exponentially tapered slot is known to favor broad bandwidth [15] but the radiation pattern is excessively wide in its E-plane. The crossed double-slot configuration improves similarity between $\mathrm{E}$ - and $\mathrm{H}$-plane radiation patterns, increasing the directivity. The crossed exponential slots alone a)

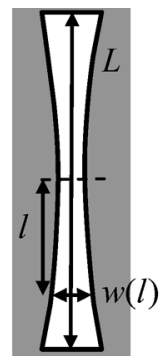

b)

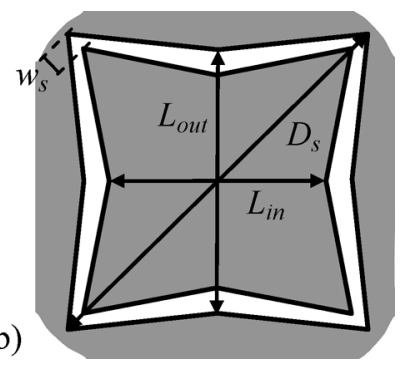

Fig. 2. Parameters defining the XETS slots: a) single exponentially tapered slot; b) star slot.

TABLE I

ANTENNA PARAMETER VALUES IN MiLlimeters

\begin{tabular}{|c|c|c|c|c|}
\hline$D_{\text {front }}$ & $D_{\text {back }}$ & $D_{s}$ & $L_{\text {out }}$ & $L_{\text {in }}$ \\
\hline 35 & 16 & 31.44 & 18.13 & 14.77 \\
\hline$w_{s}$ & $L$ & $w_{0}$ & $C_{0}$ & $h$ \\
\hline 1.57 & 22 & 0.2 & 5.96 & 0.254 \\
\hline
\end{tabular}

produce a bandwidth in the order of $50 \%$; the star slot adds an extra resonance at higher frequencies, thus enlarging further the whole bandwidth. The presence of this star slot does not influence the radiation pattern or the linear polarization purity.

The slot exponential geometry in Fig. 2(a) is given by

$$
w(l)=w_{0} \exp \left(l / C_{0}\right)
$$

where $w$ is the slot width and $l$ is the slot longitudinal coordinate measured from the centre of the slot, extending up to $l=L / 2$, the slot half length. Slot width at the centre is $w_{0}$ and $C_{0}$ is the exponential expansion parameter. The geometric parameters that define the star slot $D_{s}, w_{s}, L_{\text {in }}$ and $L_{\text {out }}$ are indicated in Fig. 2(b). The dependence of the antenna performance with these parameters was studied with the transient solver of CST Microwave Studio, based on Finite Integration Method [28]. A viable set of parameters is indicated in Table I. It is pointed out that the larger antenna dimension, $D_{\text {front }}$, is $35 \mathrm{~mm}$ (about $0.3 \lambda_{0}$ at $3.1 \mathrm{GHz}$ ); the antenna thickness is $h=10 \mathrm{mil}=0.254 \mathrm{~mm}$. Therefore, the XETS is a compact low-profile antenna solution.

A similar antenna configuration was first proposed by the authors in a different context for an integrated lens antenna feed for mm-wave applications [29], [30]. In the present paper that configuration is redefined to fulfill the UWB requirements, so this paper presents completely new information, including the transient behavior of the antenna and coupling to adjacent antenna. Onwards the antenna is referenced as the XETS, for brevity.

\section{FREQUENCY DOMAIN PERFormANCE}

The described antenna was fabricated (see Fig. 3) using Duroid 5880 substrate with permittivity $\varepsilon_{r}=2.2$, loss tangent $\tan (\delta)=0.0009$ and thickness $h=10 \mathrm{mil}=0.254 \mathrm{~mm}$. Although it is a balanced antenna configuration designed for a balanced feed, the prototype is fed directly by a small diameter $50 \Omega$ coaxial cable to make broadband measurements simpler. An EZ-47 semi-rigid coaxial cable (1.19 mm diameter) is soldered between the back petals, see Fig. 3(b). The coaxial cable excitation is duly considered in the simulation results shown in 
a)

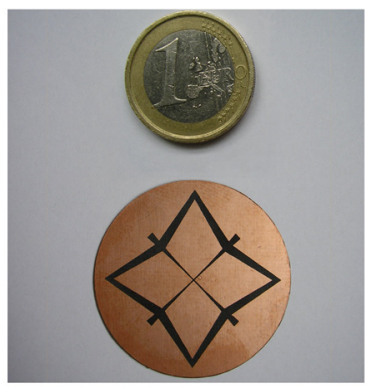

b)

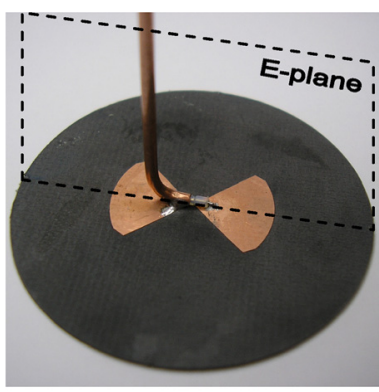

Fig. 3. Antenna prototype: a) front face view; b) back face view.

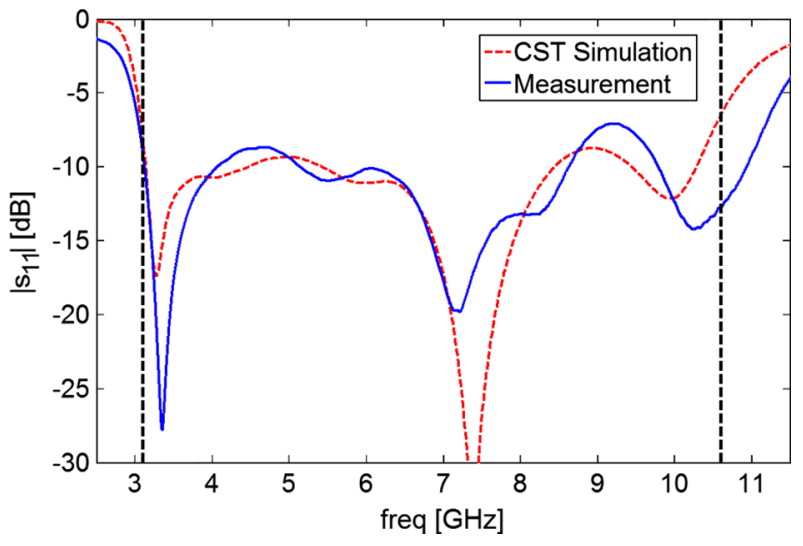

Fig. 4. Measured and simulated input reflection coefficient.

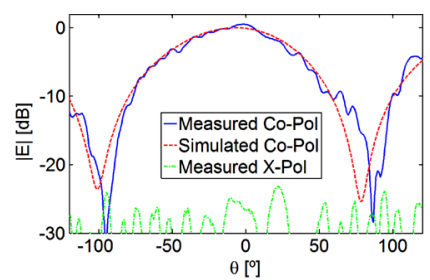

a)

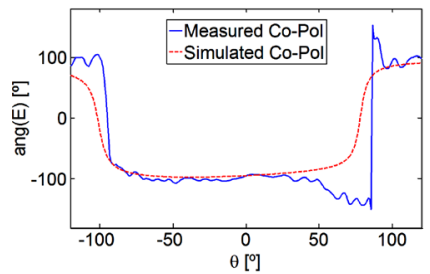

c)

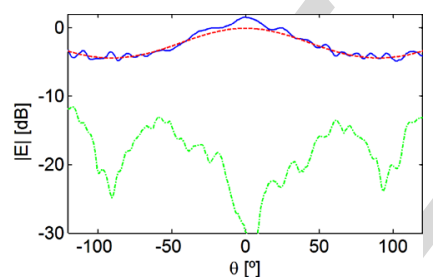

b)

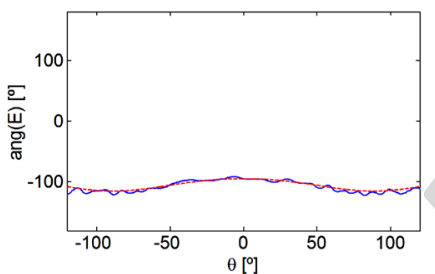

d)
Fig. 5. Measured and simulated radiation patterns at $f=4.5 \mathrm{GHz}$ : a) E-plane magnitude; b) H-plane magnitude; c) E-plane phase; d) H-plane phase.

this paper. Depending on frequency, the amplitude of the stray current flowing outside the coaxial cable is at least $10 \mathrm{~dB}$ below the current in the central conductor. Simulation comparison with balanced feeding showed that the coaxial cable produces little change in the input return loss versus frequency and only marginally degrades the antenna performance; the results shown here with coaxial feed are fully compatible with UWB system requirements, and enough to demonstrate the proposed antenna concept.

The measured input reflection coefficient of the XETS is presented in Fig. 4, which compares well with the corresponding

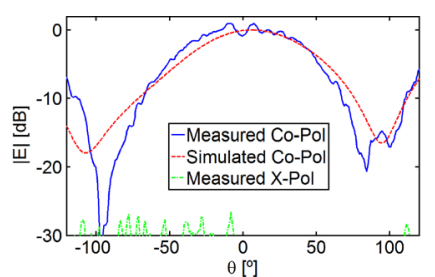

a)

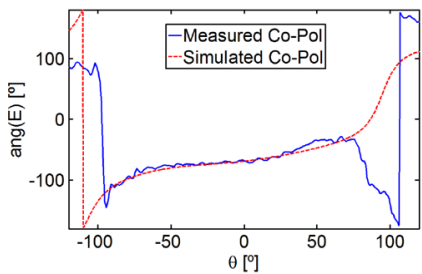

c)

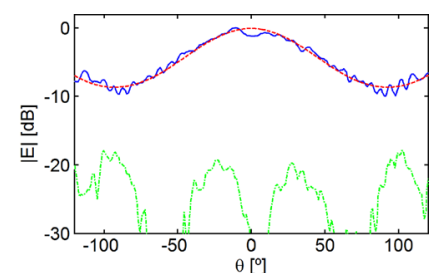

b)

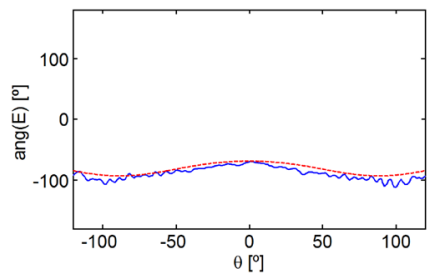

d)
Fig. 6. Measured and simulated radiation patterns at $f=7 \mathrm{GHz}$ : a) E-plane magnitude; b) H-plane magnitude; c) E-plane phase; d) H-plane phase.

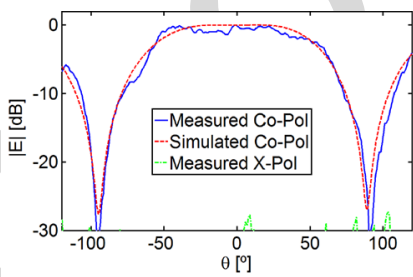

a)

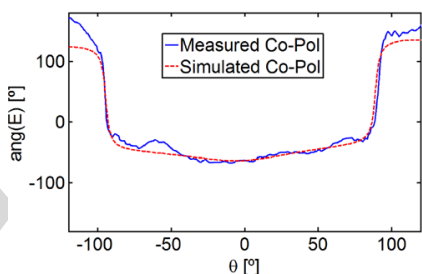

c)

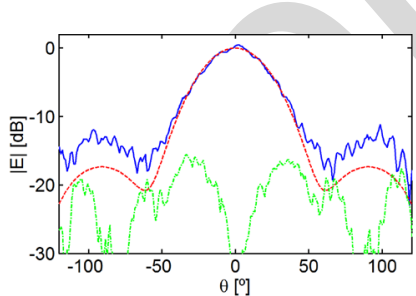

b)

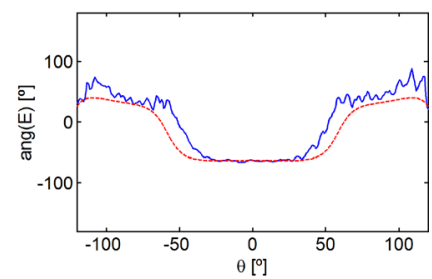

d)
Fig. 7. Measured and simulated radiation patterns at $f=9 \mathrm{GHz}$ : a) E-plane magnitude; b) H-plane magnitude; c) E-plane phase; d) H-plane phase.

CST simulated curve. A good impedance match is obtained over the entire UWB bandwidth from 3.1 to $10.6 \mathrm{GHz}$ (delimited by the two dashed vertical lines in Fig. 4) corresponding approximately to $110 \%$ bandwidth.

\section{A. Radiation Patterns}

Radiation patterns were measured for several frequencies across the band. Examples of magnitude and phase radiation pattern are shown in Figs. 5-7 for three different frequencies within the UWB band, superimposed on CST simulation curves. The $|\theta|>120^{\circ}$ region is omitted because it is blocked by the antenna tower, but the radiation pattern is bidirectional as previously referred. Part of the small ripple is originated by the reflection of the antenna back lobe on the antenna supporting tower. The excitation of the balanced antenna by the coaxial cable originates a slight lack of symmetry in the E-plane magnitude and phase radiation pattern as well as contribution to the ripple due to induced currents. Anyway the agreement with CST prediction is good, showing an almost 
constant phase characteristic which translates into a well defined constant phase centre across the elevation angle $\theta$. The measured cross-polarization is comfortably bellow $-25 \mathrm{~dB}$ in the E-plane and $-15 \mathrm{~dB}$ in the H-plane showing excellent polarization purity.

A slight increase in directivity with frequency is observed, mainly because of the narrowing of the radiation pattern in the $\mathrm{H}$-plane. However, from $8 \mathrm{GHz}$ onwards, this effect is counteracted by the broadening of the E-plane radiation pattern. CST simulations indicate that the directivity is about $4 \mathrm{dBi}$ at the lower part of the UWB band, it increases to about $6 \mathrm{dBi}$ at 8 $\mathrm{GHz}$ and then drops to about $3 \mathrm{dBi}$ at the upper end of the UWB band. These directivity results are confronted ahead with measurements.

The total antenna efficiency versus frequency, predicted by the CST model ranges from $90 \%$ to $97 \%$ across the bandwidth. This was confirmed by measurement at the lower frequencies.

\section{B. Transfer Functions}

Two equal XETS antennas are used for simulation and experimental link transfer function studies in this section. The antenna normalized vector transfer functions in transmit and receive modes $\vec{A}_{n, T x}(f, \theta, \phi)$ and $\vec{H}_{n, R x}(f, \theta, \phi)$, relate the radiated electric field to the driving antenna voltage and relate the received voltage to the incident electric field, respectively [2]. For the same antenna and following from time domain reciprocity, these functions relate as [31]

$$
A_{n, T x}=\frac{j \omega}{2 \pi c} H_{n, R x} .
$$

For equal parallel antennas, the ratio $s_{21}$ between the output voltage at the receiving antenna and the applied voltage at the transmitting antenna can be written as [13]

$$
s_{21}(f, \theta, \phi)=\frac{j \lambda}{4 \pi R}\left[H_{n, R x}^{\prime}(f, \theta, \phi)\right]^{2} \mathrm{e}^{-j 2 \pi f \frac{R}{c}}
$$

where $R$ is the distance between both antennas, and $H_{n, R x}^{\prime}=$ $H_{n, R x} \sqrt{4 \pi} / \lambda$.

Measurements of $s_{21}$ were performed in anechoic chamber using a Vector Network Analyzer (Agilent PNA E8361A) and two identical face-to-face co-polarized XETS antennas separated by $R=50 \mathrm{~cm}$. Prior full-port calibration referred to the antennas input ports was performed in order to remove frequency dependence from the RF cables. In this set-up the supporting structure was made from low-density polyurethane foam to minimize structure influence on the measurements. Fig. 8 shows the measured $S_{21}$ and the corresponding CST simulated curve, which agree quite well.

The magnitude of the transfer function is reasonably constant up to $8.5 \mathrm{GHz}$ and the unwrapped phase is almost linear over the entire UWB band which is expected to translate into low distortion of a transmitted UWB pulse. a)

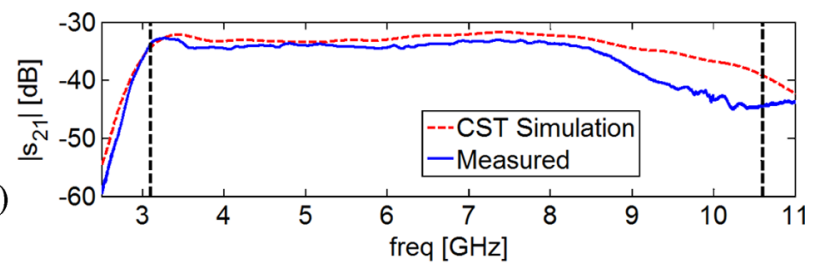

b)

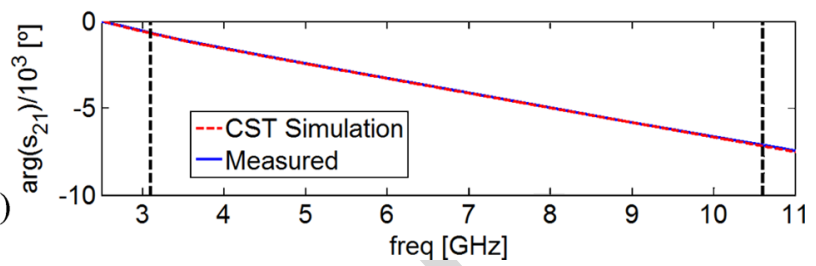

Fig. 8. Link transfer function between two identical face-to-face XETS antennas separated by $R=50 \mathrm{~cm}$ : a) amplitude; b) phase.

a)

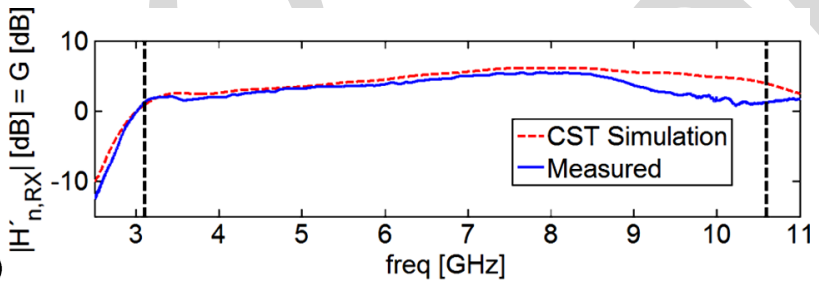

b)

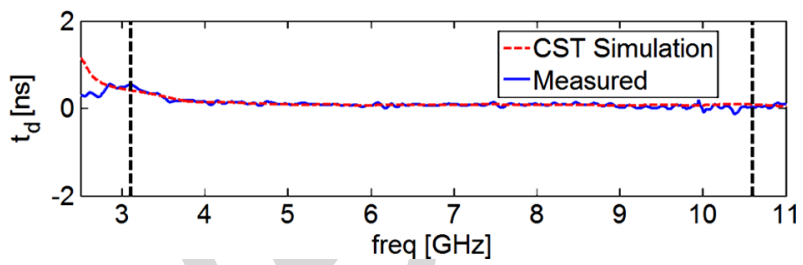

Fig. 9. a) Magnitude of $H_{n, R x}^{\prime}(\theta=\phi=0)$, which is coincident with the CW effective antenna gain in $\mathrm{dB}$ scale; b) group delay of the XETS.

Function $H_{n, R x}^{\prime}$ directly relates to continuous wave CW effective gain of the antenna [13], [31]

$$
\left|H_{n, R x}^{\prime}(f, \theta, \phi)\right|=\sqrt{G(f, \theta, \phi)} .
$$

Function $H_{n, R x}^{\prime}$ can be readily computed from (3) as a square root, and the result is shown in Fig. 9(a). It confirms the previously referred gain dependence versus frequency that was observed in the radiation patterns of Fig. 5 to Fig. 7.

Measured group delay is shown in Fig. 9(b), superimposed on CST simulated curve. The equivalent electrical length of the short feeding cable that is soldered to the antenna has been subtracted. Group delay is not only quite constant over the whole band but it is further very close to zero $(<500 \mathrm{ps})$. This means that the phase centre of the antenna remains very close to the antenna plane over the entire UWB bandwidth.

Additional measurements were performed for other $(\theta, \phi)$ antenna orientations with one XETS antenna fixed while rotating the other XETS antenna in its E- and H-plane as would be done for radiation pattern measurement. Results showed similar type 


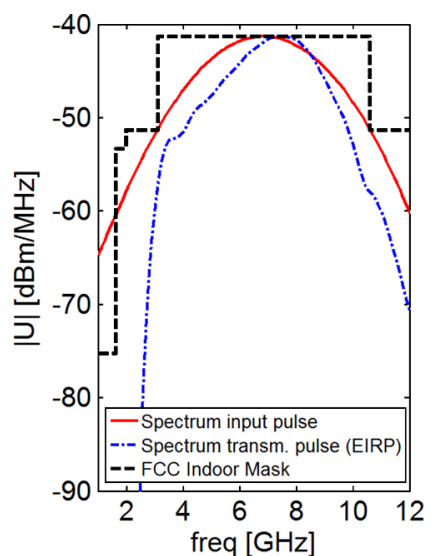

a)

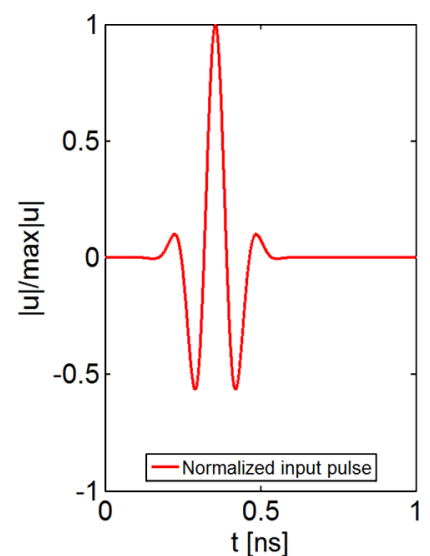

b)
Fig. 10. a) FCC indoor mask and complying spectrum of a test Gaussian pulse; b) corresponding pulse in time domain.

of frequency response and agreement with simulations. Both are presented ahead, in terms of time domain parameters.

\section{TIME DOMAIN PERFORMANCE}

\section{A. Input Pulse Characterization}

The performance of a UWB antenna can be quantified by the degree of distortion that it introduces in the transmission of a pulse. This distortion not only depends upon the antenna transfer function but also on the input waveform and the associated spectrum [32]. The test pulse adopted in the present work is defined as

$$
u(t)=\cos \left(2 \pi f_{c} t\right) \exp \left[-2 \pi(t / \tau)^{2}\right]
$$

where the central frequency is $f_{\mathrm{c}}=6.85 \mathrm{GHz}$ in order to center the pulse spectrum within the UWB band and the Gaussian width is $\tau=228$ ps to comply with the FCC indoor spectrum mask shown in Fig. 10(a). This mask limits power emission of the UWB radios to avoid possible interference with other radio systems operating within the same band [1]. The pulse amplitude in time and frequency domains is presented in Fig. 10. The entire band from 3.1 to $10.6 \mathrm{GHz}$ is used in order to produce the shortest possible pulse. In this respect, the indoor mask is more demanding to test the antenna performance than the outdoor mask. Fig. 10 also shows the transmitted pulse spectrum (EIRP at $1 \mathrm{MHz}$ bandwidth of single XETS for $\theta=\phi=0^{\circ}$ ) which fully complies with the FCC indoor mask.

The pulse defined in (5) is a good approximation of the up-converted triangular pulse which was demonstrated in [33] using CMOS technology for low-complexity, low-cost, low power consumption UWB transceivers. Simulations (not presented here) show that both pulse functions yield very similar results. Function (5) is adopted for possible comparison of the XETS results with other antenna results existing in the literature for the same excitation.

\section{B. Two-XETS Link Performance}

The measured link transfer function $s_{21}$ presented in Fig. 8, is used to obtain the shape of the received pulse at the second

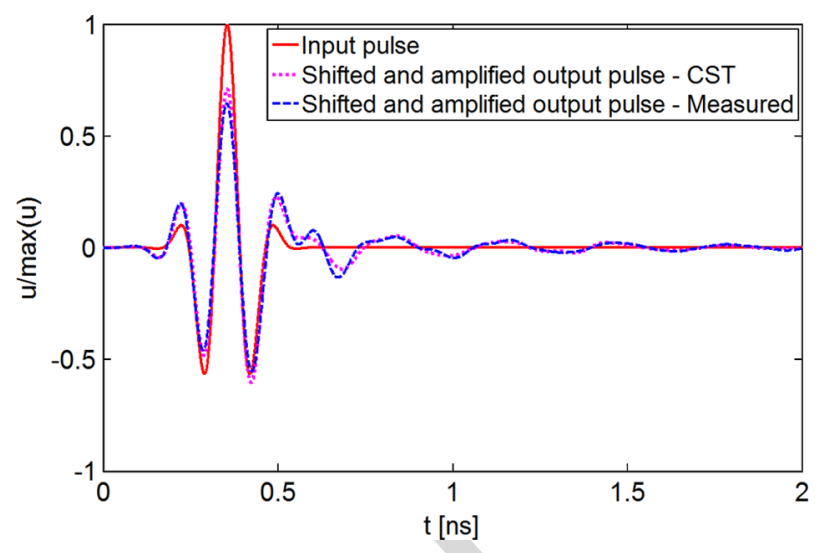

Fig. 11. Input and output pulse in time domain for a UWB link with two identical face-to-face XETS antennas.

antenna. It is represented by the dashed (blue) curve in Fig. 11 and CST simulation is superimposed (dotted magenta curve). Both curves agree quite well.

For better comparison between input and output pulses, the latter was normalized to the maximum value of $\left|s_{21}\right|$ and shifted in time. The output pulse shows good preservation of the input shape, although ringing inevitably appears at the trailing edge stretching the pulse duration. Two figures of merit can be used for more objective characterization of the output pulse distortion. One is the pulse fidelity $F(\theta, \phi)$ [2], and the other is based on the time window containing $90 \%$ of the pulse energy [3], [34], onwards referred as the $\operatorname{E} 90(\theta, \phi)$. The ratio of E90 time windows computed for the output and input pulses gives a measure of the time stretch of the output pulse introduced by the link.

For the pulse in Fig. 11, corresponding to the face-to-face two-antenna link, the measured result gives $F=93.1 \%, \mathrm{E} 90=$ $0.25 \mathrm{~ns}$, against $F=94.9 \%, \mathrm{E} 90=0.23 \mathrm{~ns}$ for the simulation prediction. For the input pulse E90 $=0.15 \mathrm{~ns}$, so the output pulse stretch ratio is 1.67 for measurements and 1.53 for simulation. The obtained values are compatible with envisaged UWB applications, allowing bit rates of hundreds of Mbit/s.

\section{Single Antenna Performance}

It would be interesting to investigate the antenna transient performance parameters over the whole solid angle, instead of just the axial direction. However this is not feasible with the used measurement setup except for the main planes. The same happens with the corresponding two-antenna CST simulation due to excessive required computational effort.

The alternative is to characterize a single antenna in the transmit mode. The distortion parameters definition now involve the detected E-field $E(t, \theta, \phi)$ pulse instead of the received voltage pulse in the receive antenna. It is clear that distortion parameter values obtained for a single XETS are different from those obtained for the two-antenna link, yet they are also meaningful and important.

In CST simulation this analysis can be done using a far-field linear polarized electrical field probe over the antenna solid angle [28]. In the experimental set-up, the spectrum of the transmitted E-field at an observation point at distance $R$ can be 


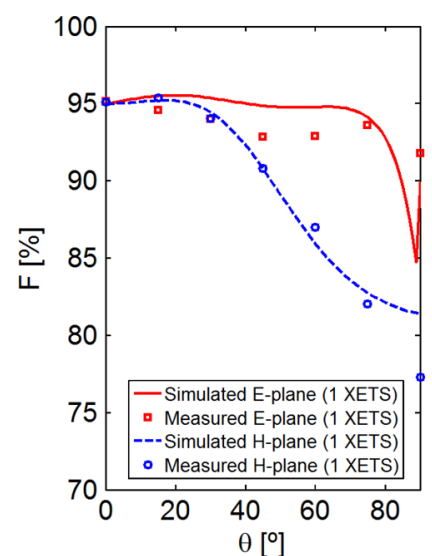

a)

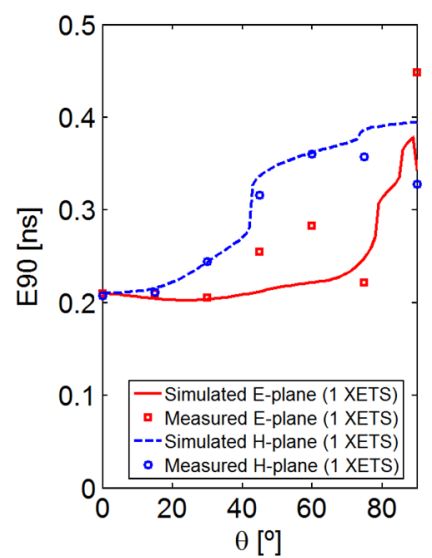

b)
Fig. 12. Output pulse distortion parameters for a single XETS antenna. a) Fidelity; b) E90 time window (the E90 window for the input pulse is $0.15 \mathrm{~ns}$ ).

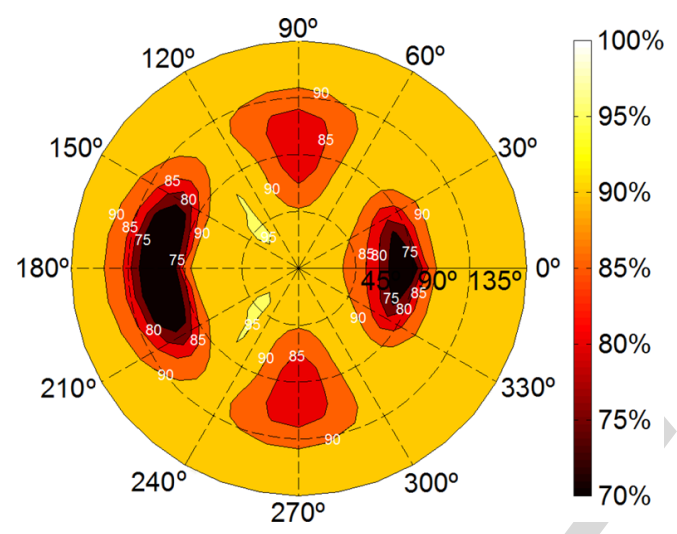

Fig. 13. Fidelity of a single XETS antenna over the soli angle. The radial angle is theta and the polar angle is phi.

obtained from the measured transfer function of the two-XETS link [2]

$$
\frac{E(f, R, \theta, \phi)}{\sqrt{120 \pi}}=\frac{j}{\sqrt{4 \pi} R} \frac{U(f)}{\sqrt{Z_{\text {in }}}} H_{n, R x}^{\prime}(f, \theta, \phi) \mathrm{e}^{-j 2 \pi f \frac{R}{c}}
$$

where $Z_{\text {in }}$ is the transmitting antenna characteristic port impedance and $U(f)$ is the spectrum of the applied voltage.

Measured and simulated values of the pulse distortion parameters are shown in Fig. 12 for the E- and H-planes of a single XETS antenna. Fidelity values range from $77.3 \%$ to $95.4 \%$ and E90 time window range from $0.21 \mathrm{~ns}$ to $0.45 \mathrm{~ns}$.

It is noted that the agreement between simulation and measurement is good, which allows exploring further the antenna properties using CST simulations. Fig. 13 presents the calculated fidelity of a single XETS antenna over the whole solid angle. The value ranges from $70 \%$ in the $\mathrm{E}$ - and $\mathrm{H}$-planes near $\theta=90^{\circ}$, to $95 \%$ in most of the front and back hemispheres. The $F$ drop close to $\theta=90^{\circ}$ is originated by the XETS radiation null in that direction; see Fig. 5(a) to Fig. 7(a). The $F$ drop is less abrupt in the H-plane close to $\theta=90^{\circ}$ because the radiation level is higher in this direction, see Fig. 5(b) to Fig. 7(b). The slight lack of overall symmetry is due to the feeding coaxial cable.

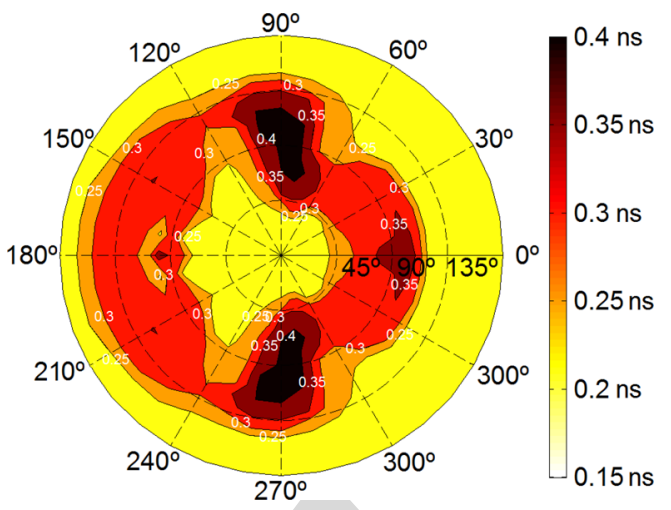

Fig. 14. Time window containing $90 \%$ of the pulse energy transmitted by a XETS antenna over the solid angle. The radial angle is theta and the polar angle is phi (the E90 window for the input pulse is $0.15 \mathrm{~ns}$ ).

The E90 time window results are presented in Fig. 14. The values range from $0.21 \mathrm{~ns}$ in the front and back directions to $0.41 \mathrm{~ns}$ in the $\mathrm{H}$-plane close to the $\theta=90^{\circ}$. However, while in the E-plane the larger pulse stretch is confined around $\theta=90^{\circ}$, the same does not happen in the H-plane. This is a consequence of the stronger frequency dependence of the H-plane radiation pattern. It is also relevant to point out that the maximum E90 time window of a single XETS antenna in the axial direction is only $0.21 \mathrm{~ns}$ while in the simulated complete radio link (Fig. 11) it is $0.23 \mathrm{~ns}$; in the latter case two antennas contribute for the pulse distortion.

So, safe for a little drop at the plane that contains the antenna, $F$ and E90 values are very good and stable over the solid angle, showing low pulse distortion.

\section{MultiAntenna ARrangements}

It was mentioned in the introduction that complementary steps for UWB radio development are related with the combination of this technology with the diversity and even with multiple-input-multiple-output (MIMO) concepts to enhance transmission data-rates and range [22], [23]. Spatial and polarization diversity can be explored in the above context; this requires good isolation between adjacent antennas, and between polarization diversity channels. This type of study on the UWB antenna part is just starting to appear in the literature [35].

The proposed XETS antenna presents excellent characteristics in this respect. The back petals shown in Fig. 1 for attaching the excitation to the XETS antenna can be repeated also for the orthogonal pair of petals to produce an almost independent orthogonal polarization mode in the same antenna. This configuration constitutes a solution of its own and it is not addressed here. Instead, this section quantifies the coupling between adjacent antennas in the E-plane, H-plane and mixed E- and H-planes (see Fig. 15). This coupling is evaluated trough the $s_{21}$ parameter across the UWB band and through the ratio $\varepsilon$ between the energy contained in the pulse received in one antenna to the energy transmitted in the adjacent antenna.

In this characterization the adjacent antennas are coplanar, printed on a common substrate and its centers are separated by the minimum distance $D_{\text {front }}$, that is $35 \mathrm{~mm}\left(0.3 \lambda_{0}\right)$. The results are summarized in Fig. 16. It is clear that the crossed 

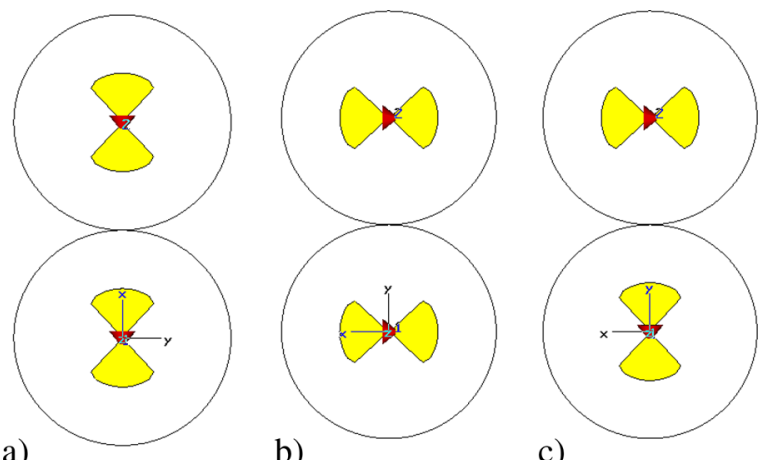

c)

Fig. 15. Double-antenna arrangement: a) E-plane; b) H-plane; c) crossed arrangement.

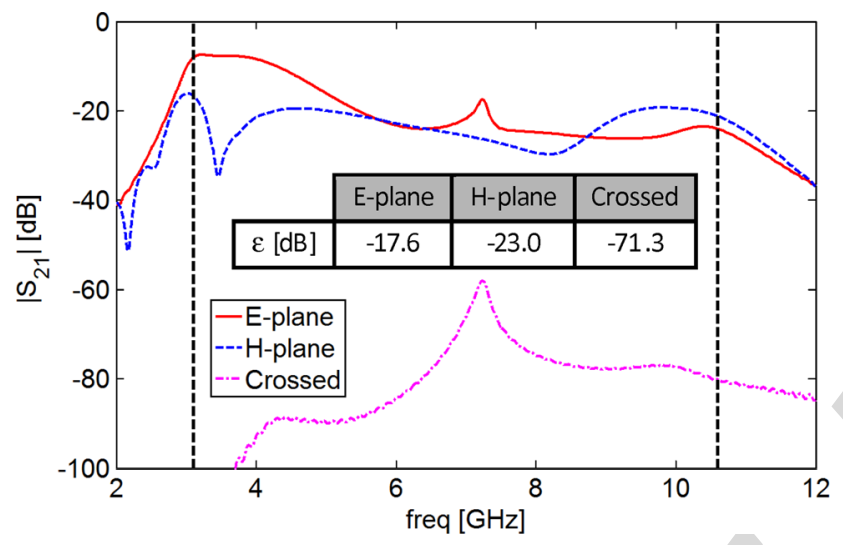

Fig. 16. Coupling between two XETS antennas in the different double arrangements. The inset table presents the ratio between the energy in the received pulse in one antenna to the energy transmitted by the adjacent antenna.

arrangement yields the best isolation, but even the H-plane arrangement is quite satisfactory. It should be mentioned that the good H-plane isolation for a similar antenna in a different context (mm-wave lens feed array) has been confirmed experimentally [30].

Time domain simulations have further shown that, any of the tested double-antenna arrangements in Fig. 15 produces little change in the $F$ and E90 time window compared to the isolated antenna case (calculations based on transmit mode transfer function obtained with field probe).

\section{CONCLUSION}

The presented study confirmed with measurements shows that the XETS is a very good antenna candidate for UWB communications using the FCC assigned 3.1-10.6 GHz. The antenna diameter is $35 \mathrm{~mm}\left(0.3 \lambda_{0}\right.$ at $\left.3.1 \mathrm{GHz}\right)$ and substrate is 10 mil thick; so the antenna is compact and low-profile. Owing to the crossed exponential slots and to the antenna perfect two-plane symmetry, the radiation pattern presents smooth dependence with frequency and its linear polarization is shown to be completely stable for all observation angles, with low cross-polarization level and low mutual coupling in multi-antenna arrangements. This characteristic is not so common in other reported antenna solutions. It makes the XETS especially attractive for combining the UWB concept with diversity and
MIMO strategies to enhance link capacity and improve range, which is constrained by the FCC power emission mask for UWB. The balanced antenna configuration is adequate for direct integration of differential UWB RFICs at the back of the antenna, avoiding the need for an additional balun, thus decreasing the complexity of the UWB transceiver.

Time domain characterization of the XETS showed as well low pulse distortion effects, thoroughly quantified through the pulse fidelity parameter (ranging from $70 \%$ to $95 \%$ across the solid angle) and by the width of the $90 \%$ energy window (ranging from $0.21 \mathrm{~ns}$ to $0.41 \mathrm{~ns}$ ). The worse results correspond to the plane containing the printed antenna, which is unavoidable. These results are compatible with the envisaged several hundred Mbit/s data rates. Improved results would be obtained with a balanced feed.

It is interesting to mention that the proposed antenna configuration still leaves room for further developments. The addition of appropriately designed slots in the XETS can produce a notch in the antenna transfer function to prevent interference with WLAN bands and avoid extra dedicated filtering components in the system. These results will appear elsewhere. Other line for further developments is the duplication of the back-face petals to enable dual polarization excitation of the same XETS for achieving a very compact polarization diversity arrangement.

\section{ACKNOWLEDGMENT}

The authors acknowledge the collaboration from V. Fred and C. Brito for prototype construction, and A. Almeida for prototype measurements.

\section{REFERENCES}

[1] Federal Communications Commission (FCC), First Order and Report: Revision of Part 15 of the Commission's Rules Regarding UWB Transmission Systems FCC 02-48, Apr. 22, 2002.

[2] D. Lamensdorf and L. Susman, "Baseband-pulse-antenna techniques," IEEE Antennas Propag. Mag., vol. 36, pp. 20-30, Feb. 1994.

[3] M. Klemm and G. Tröster, "Characterization of small planar antennas for UWB mobile terminals," Wireless Commun. Mobile Comput., vol. 5, no. 5, pp. 525-536, Aug. 2005.

[4] Z. Chen et al., "Planar antennas," IEEE Microw. Mag., vol. 7, no. 6, pp. 63-73, Dec. 2006.

[5] T. Karacolak and E. Topsakal, "A double-sided rounded bow-tie antenna (DSRBA) for UWB communication," IEEE Antennas Wireless Propag. Lett., vol. 5, pp. 446-449, 2006.

[6] X. Wu, Z. Chen, and N. Yang, "Optimization of planar diamond antenna for single-band and multiband UWB wireless communications,' Microw. Opt. Technol. Lett., vol. 42, no. 6, pp. 451-455, Sept. 2004.

[7] J. Zhang, Y. Xu, and W. Wang, "Ultra-wideband microstrip-fed planar elliptical dipole antenna," Electron. Lett., vol. 42, no. 3, pp. 144-145, Feb. 2006.

[8] E. Gueguen, F. Thudor, and P. Chambelin, "A low cost UWB printed dipole antenna with high performance," in Proc. IEEE Inter. Conf. on Ultra-Wideband-ICU 2005, Zurich, Switzerland, Sep. 2005, pp. 89-92.

[9] S. Zhong, X. Liang, and W. Wang, "Compact elliptical monopole antenna with impedance bandwidth in excess of 21:1," IEEE Trans. Antennas Propag., vol. 55, no. 11, pp. 3080-3085, Nov. 2007.

[10] J. Liang et al., "Study of a printed circular disc monopole antenna for UWB systems," IEEE Trans. Antennas Propag., vol. 53, no. 11, pp. 3500-3504, Nov. 2005.

[11] A. Mohamed and L. Shafai, "Investigation on the phase centre of ultra wideband circular monopole antennas," presented at the IEEE Antennas Propag. Society Inter. Symp., San Diego, CA, Jul. 2008.

[12] W. Lui, C. Cheng, and H. Zhu, "Improved frequency notched ultrawideband slot antenna using square ring resonator," IEEE Trans. Antennas Propag., vol. 55, no. 9, pp. 2445-2450, Sep. 2007. 
[13] T. Ma and S. Jeng, "Planar miniature tapered-slot-fed annular slot antennas for ultrawideband radios," IEEE Trans. Antennas Propag., vol. 53, no. 3, pp. 1194-1202, Mar. 2005.

[14] I. J. Yoon et al., "Ultrawideband tapered slot antenna with band cutoff characteristic," Electron. Lett., vol. 41, no. 11, pp. 629-630, May 2005.

[15] C. Chen, W. McKinzie, and N. Alexopoulos, "Stripline-fed arbitrarily shaped printed-aperture antennas," IEEE Trans. Antennas Propag., vol. 45, no. 7, pp. 1186-1198, Jul. 1997.

[16] J. Martínez-Fernández, J. Gil, and J. Zapata, "Ultrawideband optimized profile monopole antenna by means of simulated annealing algorithm and the finite element method," IEEE Trans. Antennas Propag., vol. 55, no. 6, pp. 1826-1832, Jun. 2007.

[17] M. Ammann, R. Cordoba, M. Uzelac, J. Evans, and A. Schwarzbacher, "On pattern stability of the crossed planar monopole," Microw. Opt. Technol. Lett., vol. 40, no. 4, pp. 294-296, Feb. 2004.

[18] X. Li, S. Hagness, M. Choi, and D. van der Weide, "Numerical and experimental investigation of an ultrawideband ridged pyramidal horn antenna with curved launching plane for pulse radiation," IEEE Antennas Wireless Propag. Lett., vol. 2, pp. 259-262, 2003.

[19] J. Andrews, UWB Signal Sources, Antennas and Propagation Picosecond Pulse Labs, Application Note AN-30414a, pp. 1-11, Aug. 2003.

[20] M. Blech and T. Eibert, "A dipole excited ultrawideband dielectric rod antenna with reflector," IEEE Trans. Antennas Propag., vol. 55, no. 7, pp. 1948-1954, Jul. 2007.

[21] S. Bruni, A. Neto, and F. Marliani, "The ultrawideband leaky lens antenna," IEEE Trans. Antennas Propag., vol. 55, no. 10, pp. 2642-2653, Oct. 2007.

[22] W. Malik and D. Edwards, "Measured MIMO capacity and diversity gain with spatial and polar arrays in ultrawideband channels," IEEE Trans. Commun., vol. 55, no. 12, pp. 2361-2370, Dec. 2007.

[23] J. Keignart, C. Abou-Rjeily, C. Delaveaud, and N. Daniele, "UWB SIMO channel measurements and simulations," IEEE Trans. Microw. Theory Tech., vol. 54, no. 4, pp. 1812-1819, Jun. 2006.

[24] A. Vorobyov et al., "Integration of a pulse generator on chip into a compact ultrawideband antenna," IEEE Trans. Antennas Propag., vol. 56, no. 3, pp. 858-868, Mar. 2008.

[25] Agilent-Ultra-Wideband Communication RF Measurements Application Note 1488, 2005.

[26] P. Datta, X. Fan, and G. Fischer, "A transceiver front-end for ultrawide-band applications," IEEE Trans. Circuits Systems-II: Express Briefs, vol. 54, no. 4, pp. 362-366, Apr. 2007.

[27] J. Costa and C. Fernandes, "Crossed exponentially tapered slot antenna for UWB applications," presented at the IEEE Antennas Propag. Society Inter. Symp., San Diego, CA, Jul. 2008.

[28] CST [Online]. Available: www.cst.com

[29] J. Costa and C. Fernandes, "Broadband slot feed for integrated lens antennas," IEEE Antennas Wireless Propag. Lett., vol. 6, pp. 396-400, 2007.

[30] J. Costa and C. Fernandes, "Integrated imaging lens antenna with broadband feeds," presented at the 2nd Eur. Conf. on Antennas Propag.-EUCAP, Edinburgh, U.K., Nov. 2007.

[31] C. Baum, E. Farr, and C. Frost, "Transient gain of antennas related to the traditional continuous-wave (CW) definition of gain," in Proc. Ultra-Wideband Short-Pulse Electromagn., Jun. 1998, vol. 4, pp. 109-118.

[32] D. Pozar, "Waveform optimizations for ultrawideband radio systems," IEEE Trans. Antennas Propag., vol. 51, no. 9, pp. 2335-2345, Sep. 2003.

[33] J. Ryckaert, "Ultrawideband transmitter for low-power wireless body area networks: Design and evaluation," IEEE Trans. Circuits Syst., vol. 52, no. 12, pp. 2515-2525, Dec. 2005.
[34] N. Fortino, J.-Y. Dauvignac, G. Kossiavas, and R. Staraj, "Design optimization of UWB printed antenna for omnidirectional pulse radiation," IEEE Trans. Antennas Propag., vol. 56, no. 7, pp. 1875-1881, Jul. 2008.

[35] Z. Chen, "UWB antennas with enhanced performances," in Proc. Int. Conf. on Microw. and Millimeter Wave Technology, ICMMT 2008, Nanjing, China, Apr. 2008, vol. 1, pp. 387-390.

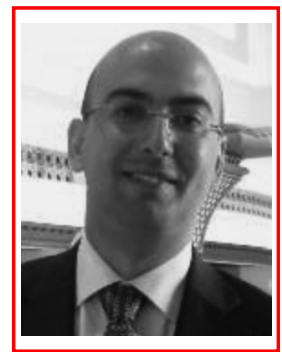

Jorge R. Costa (S'97-M'03) was born in Lisbon, Portugal, in 1974. He received the Licenciado and $\mathrm{Ph} . D$. degrees in electrical and computer engineering from the Instituto Superior Técnico (IST), Technical University of Lisbon, Lisbon, Portugal, in 1997 and 2002 , respectively.

$\mathrm{He}$ is currently a Researcher at the Instituto de Telecomunicações, Lisbon, Portugal. He is also an Assistant Professor at the Departamento de Ciências e Tecnologias da Informação, Instituto Superior de Ciências do Trabalho e da Empresa. His present research interests include lenses, reconfigurable antennas, MEMS switches, UWB and RFID antennas.

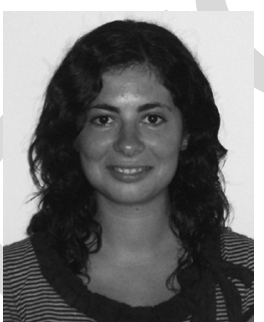

Carla R. Medeiros was born in Ponta Delgada, Açores, Portugal, in 1982. She received the Licenciado and M.Sc. degrees in electrical and computer engineering from the Instituto Superior Técnico (IST), Technical University of Lisbon, Lisbon, Portugal, in 2006 and 2007, respectively.

Since 2006, she has been a researcher at the Instituto de Telecomunicações (IT), focusing her work on antenna for wireless communications. She collaborates in national research projects. Her current research interests are in the areas of reconfigurable,

RFID and UWB antennas.

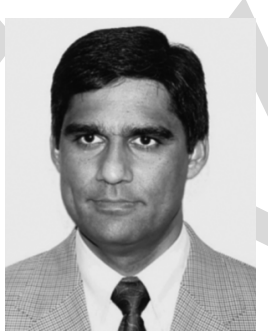

Carlos A. Fernandes (S'86-M'89-SM'08) received the Licenciado, M.Sc., and Ph.D. degrees in electrical and computer engineering from Instituto Superior Técnico (IST), Technical University of Lisbon, Lisbon, Portugal, in 1980, 1985, and 1990, respectively.

He joined the IST in 1980, where he is presently Full Professor at the Department of Electrical and Computer Engineering in the areas of microwaves, radio wave propagation and antennas. He is a Senior Researcher at the Instituto de Telecomunicações and Coordinator of its Wireless Communications scientific area. He has been the leader of antenna activities in National and European Projects as RACE 2067-MBS (Mobile Broadband System), ACTS AC230 - SAMBA (System for Advanced Mobile Broadband Applications) and ESA/ESTEC-ILASH (Integrated Lens Antenna Shaping). He has coauthored a book, a book chapter, and more than 100 technical papers in international journals and conference proceedings, in the areas of antennas and radiowave propagation modeling. His current research interests include dielectric antennas for millimeter wave applications, antennas and propagation modeling for personal communication systems, RFID antennas, artificial dielectrics and metamaterials. 


\title{
Performance of a Crossed Exponentially Tapered Slot Antenna for UWB Systems
}

\author{
Jorge R. Costa, Member, IEEE, Carla R. Medeiros, and Carlos A. Fernandes, Senior Member, IEEE
}

\begin{abstract}
A compact printed antenna is described that exhibits adequate transient performance for ultrawideband (UWB) applications and it is further adequate for polarization diversity schemes. The antenna is based on an original combination of two crossed exponentially tapered slots plus a star-shaped slot to produce a stable radiation pattern with very stable polarization over the 3.1-10.6 GHz FCC assigned band. Results are confirmed with measurements. Figures of merit like output pulse fidelity and time window containing $90 \%$ of the transmitted energy are analyzed over the entire solid angle and showed to remain quite stable, in line with envisaged UWB system requirements. Compact dual-antenna arrangements are also analyzed in view of potential use for UWB multiple-input-multiple-output implementations.
\end{abstract}

Index Terms-Antenna transient analysis, linear polarization, printed broadband antenna, tapered slot antenna, ultrawideband (UWB) antennas.

\section{INTRODUCTION}

$\mathbf{U}$ LTRAWIDEBAND (UWB) technology has been receiving increased interest as a promising solution for extremely high data rate short-range wireless indoor applications. The operation mode of these radio systems is based on transmission/reception of trains of very short pulses, which require a large bandwidth. In the United States, the Federal Communications Commission FCC [1] authorized the unlicensed use of the $3.1-10.6 \mathrm{GHz}$ spectrum for UWB applications with limited power spectral density emission to enable spectrum sharing with other established narrowband applications without mutual interference.

Antennas play a critical role in the UWB radio performance, since they influence the shape of the output pulse, acting as a band-pass filter both in frequency and in spatial domains. So, further to the classical frequency domain analysis of antenna impedance match, polarization, gain and radiation pattern over the UWB band, also the antenna pulse-preserving capability must be appropriately considered in the antenna design. The latter can be evaluated through two figures of merit: the fidelity factor [2] and the pulse stretch ratio [3].

Manuscript received August 25, 2008; revised December 05, 2008.

J. R. Costa is with Instituto de Telecomunicações, IST, 1049-001 Lisboa, Portugal and also with the Instituto Superior de Ciências do Trabalho e da Empresa, Departamento de Ciências e Tecnologias da Informação, 1649-026 Lisboa, Portugal (e-mail: Jorge.Costa@1x.it.pt).

C. R. Medeiros and C. A. Fernandes are with the Instituto de Telecomunicações, IST, 1049-001 Lisboa, Portugal.

Color versions of one or more of the figures in this paper are available online at http://ieeexplore.ieee.org.

Digital Object Identifier 10.1109/TAP.2009.2016727
Plenty of antenna solutions are proposed in the literature, seeking for the best compromise between performance and desirable compactness, low-profile and low-cost objectives. Printed antennas are the most popular choice to come near the above requirements, with especial interest for those configurations where the radiating element and the ground plane are co-planar or at least are printed on each side of a single dielectric substrate [4]. Most of these planar UWB antenna solutions can be catalogued into two broad groups: dipole/monopole-like and slot-based antennas.

Examples of wideband planar dipole type antennas include bow tie, diamond, elliptical and circular antenna [5]-[8]. But a larger number of solutions are based on planar monopole antennas [3], [9]-[11]. These monopoles and dipoles can provide extremely wide bandwidth with ratios of up to 1:20. However, the radiation patterns tend to present strong variation with frequency, in most cases with high level of cross-polarization and shifting phase centre position [11]. This has negative impact on pulse shape stability over the solid angle, which is a most important requirement for UWB applications.

Planar slot-based antennas are a competing approach for UWB applications [12], [13]. Although, some designs also tend to present radiation pattern stability and cross-polarization problems, tapered slot configurations [14], [15] have the advantage of usually producing a smooth bidirectional patch-like radiation pattern with low cross-polarization.

Many other non-planar solutions are also reported, including vertical monopoles with ground plane [16], [17], horn-based antennas [18], [19] and volume dielectric antennas [20], [21] for increased directivity. Some of these solutions bare the same radiation stability issues versus frequency in the solid angle of observation, but bulk is the main drawback.

A large number of reported UWB antennas are designed and optimized without paying attention to the transient response. Also polarization stability is generally disregarded considering it of little relevance, especially for personal terminal communications. However, diversity schemes are being considered to enhance UWB radio performance [22], [23]; so issues like mutual coupling in multi-antenna systems and polarization purity become relevant in this case.

The present paper proposes and evaluates the feasibility of a new UWB antenna complying with the above requirements. It is a printed planar slot-based antenna, with double-symmetry and balanced configuration. The operation bandwidth covers the whole 3.1 to $10.6 \mathrm{GHz}$ UWB band. The antenna is very compact $\left(0.3 \lambda_{0} \times 0.3 \lambda_{0}\right)$, low profile, with very pure stable linear polarization, low pulse distortion and negligible coupling to adjacent antennas in multi-antenna assemblies. The proposed balanced 

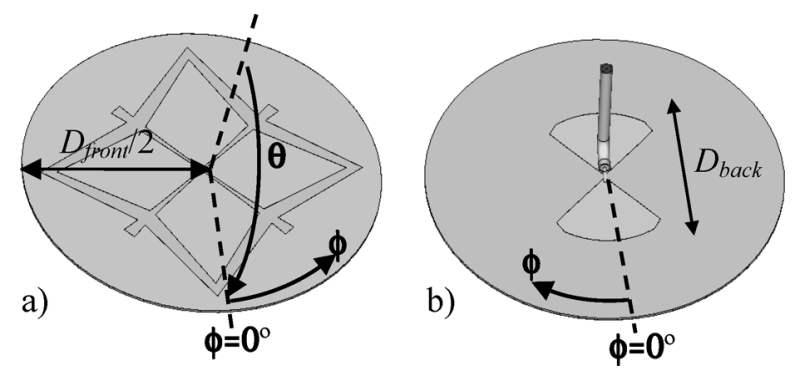

Fig. 1. XETS antenna geometry in CST Microwave Studio [28] simulation model: a) front face; b) back face.

antenna configuration is adequate for direct integration of differential RFICs at its back, without RF cables or additional balun [24]. UWB chip transceivers design based on differential signal paths are becoming standard practice [25], [26] so the balanced antenna configuration is the preferred choice in this case.

A detailed study of the antenna frequency domain characteristics and impulse response is carried out in this paper. Simulated and measured results are presented which confirm the potential of the proposed antenna for UWB systems. A feasibility study of the proposed antenna configuration, based just on simulations, was presented in [27] for a different 1:3 frequency interval.

This paper is organized as follows. The antenna concept is presented in Section II. The antenna performance is presented in Sections III for the frequency domain and in Section IV for time domain. Mutual coupling in multi-antenna arrangements is addressed in Section V. Numerical and experimental results are given and discussed throughout. Conclusions are finally drawn in Section VI.

\section{ANTENNA DESCRIPTION}

The antenna geometry is presented in Fig. 1. The front face is composed of a circular metal layer with two crossed exponentially tapered slots, intersected by a star like slot. The metal layer circumference has diameter $D_{\text {front }}$. Chopped replicas of two opposing "petals" of the antenna are printed at the back-side of the substrate, Fig. 1(b). The chopped edge of these petals is an arc of circumference with diameter $D_{\text {back }}$. The antenna is fed between the two back petals, which couple capacitively at RF with the corresponding front petals of the antenna. The $\phi=0$ plane as shown in Fig. 1 defines the antenna E-plane. It passes through the petals that are fed by the coaxial cable. The $\phi=90^{\circ}$ plane defines the antenna H-plane.

The antenna perfect symmetry with respect to feed point ensures perfect symmetry of the antenna currents with respect to the E-plane and perfect anti-symmetry with respect to the H-plane, irrespective to frequency. Consequently, pure linear polarization versus frequency is obtained at least in the main planes. The slots in the front face metallization determine the radiation pattern, which is bidirectional due to the absence of a ground plane. A single exponentially tapered slot is known to favor broad bandwidth [15] but the radiation pattern is excessively wide in its E-plane. The crossed double-slot configuration improves similarity between $\mathrm{E}$ - and $\mathrm{H}$-plane radiation patterns, increasing the directivity. The crossed exponential slots alone a)

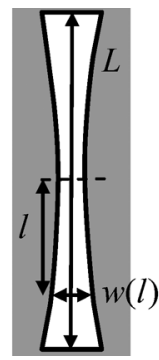

b)

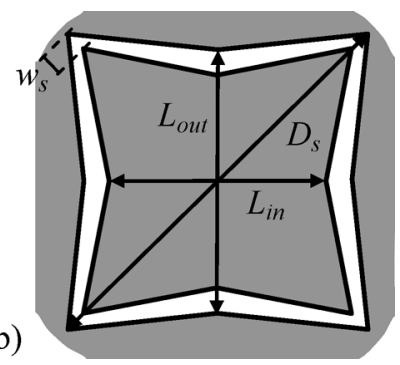

Fig. 2. Parameters defining the XETS slots: a) single exponentially tapered slot; b) star slot.

TABLE I

ANTENNA PARAMETER VALUES IN MiLlimeters

\begin{tabular}{|c|c|c|c|c|}
\hline$D_{\text {front }}$ & $D_{\text {back }}$ & $D_{s}$ & $L_{\text {out }}$ & $L_{\text {in }}$ \\
\hline 35 & 16 & 31.44 & 18.13 & 14.77 \\
\hline$w_{s}$ & $L$ & $w_{0}$ & $C_{0}$ & $h$ \\
\hline 1.57 & 22 & 0.2 & 5.96 & 0.254 \\
\hline
\end{tabular}

produce a bandwidth in the order of $50 \%$; the star slot adds an extra resonance at higher frequencies, thus enlarging further the whole bandwidth. The presence of this star slot does not influence the radiation pattern or the linear polarization purity.

The slot exponential geometry in Fig. 2(a) is given by

$$
w(l)=w_{0} \exp \left(l / C_{0}\right)
$$

where $w$ is the slot width and $l$ is the slot longitudinal coordinate measured from the centre of the slot, extending up to $l=L / 2$, the slot half length. Slot width at the centre is $w_{0}$ and $C_{0}$ is the exponential expansion parameter. The geometric parameters that define the star slot $D_{s}, w_{s}, L_{\text {in }}$ and $L_{\text {out }}$ are indicated in Fig. 2(b). The dependence of the antenna performance with these parameters was studied with the transient solver of CST Microwave Studio, based on Finite Integration Method [28]. A viable set of parameters is indicated in Table I. It is pointed out that the larger antenna dimension, $D_{\text {front }}$, is $35 \mathrm{~mm}$ (about $0.3 \lambda_{0}$ at $3.1 \mathrm{GHz}$ ); the antenna thickness is $h=10 \mathrm{mil}=0.254 \mathrm{~mm}$. Therefore, the XETS is a compact low-profile antenna solution.

A similar antenna configuration was first proposed by the authors in a different context for an integrated lens antenna feed for mm-wave applications [29], [30]. In the present paper that configuration is redefined to fulfill the UWB requirements, so this paper presents completely new information, including the transient behavior of the antenna and coupling to adjacent antenna. Onwards the antenna is referenced as the XETS, for brevity.

\section{FREQUENCY DOMAIN PERFormANCE}

The described antenna was fabricated (see Fig. 3) using Duroid 5880 substrate with permittivity $\varepsilon_{r}=2.2$, loss tangent $\tan (\delta)=0.0009$ and thickness $h=10 \mathrm{mil}=0.254 \mathrm{~mm}$. Although it is a balanced antenna configuration designed for a balanced feed, the prototype is fed directly by a small diameter $50 \Omega$ coaxial cable to make broadband measurements simpler. An EZ-47 semi-rigid coaxial cable (1.19 mm diameter) is soldered between the back petals, see Fig. 3(b). The coaxial cable excitation is duly considered in the simulation results shown in 
a)

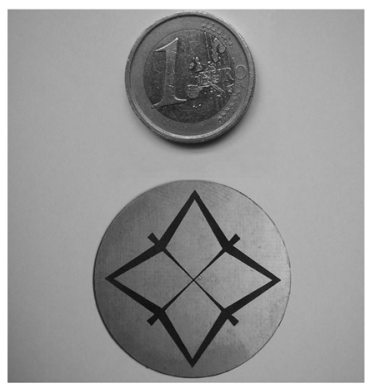

b)

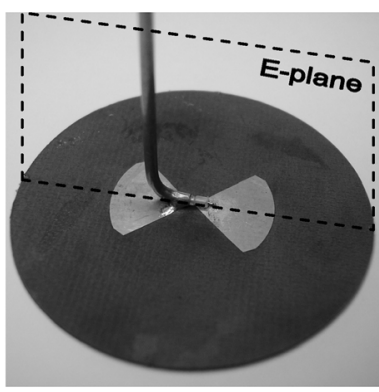

Fig. 3. Antenna prototype: a) front face view; b) back face view.

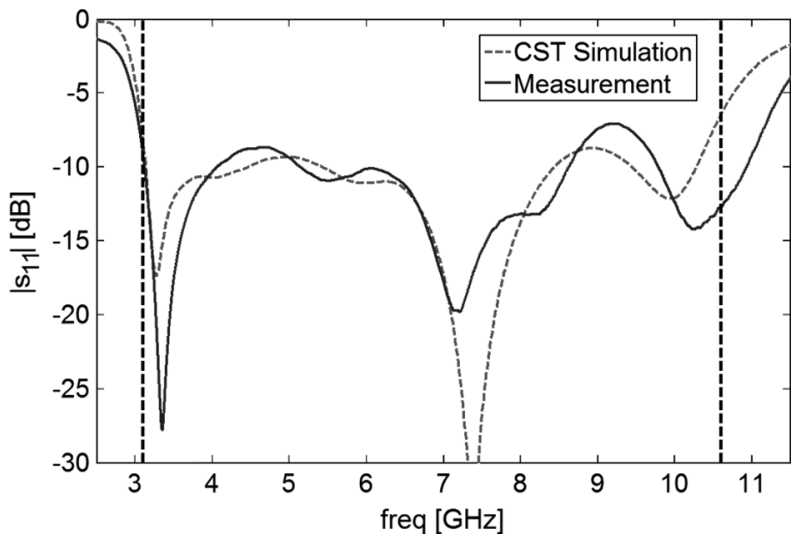

Fig. 4. Measured and simulated input reflection coefficient.

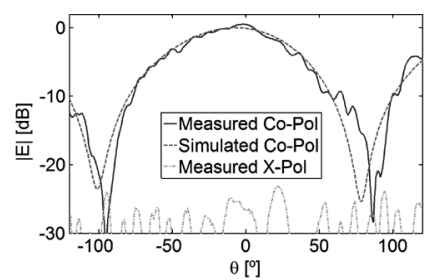

a)

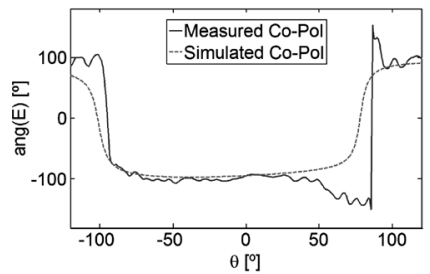

c)

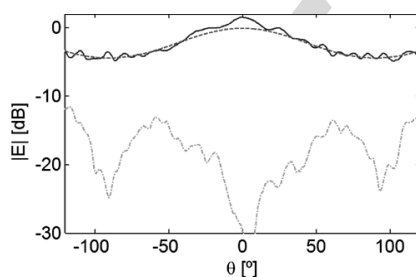

b)

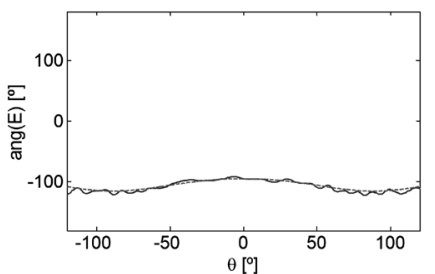

d)
Fig. 5. Measured and simulated radiation patterns at $f=4.5 \mathrm{GHz}$ : a) E-plane magnitude; b) H-plane magnitude; c) E-plane phase; d) H-plane phase.

this paper. Depending on frequency, the amplitude of the stray current flowing outside the coaxial cable is at least $10 \mathrm{~dB}$ below the current in the central conductor. Simulation comparison with balanced feeding showed that the coaxial cable produces little change in the input return loss versus frequency and only marginally degrades the antenna performance; the results shown here with coaxial feed are fully compatible with UWB system requirements, and enough to demonstrate the proposed antenna concept.

The measured input reflection coefficient of the XETS is presented in Fig. 4, which compares well with the corresponding

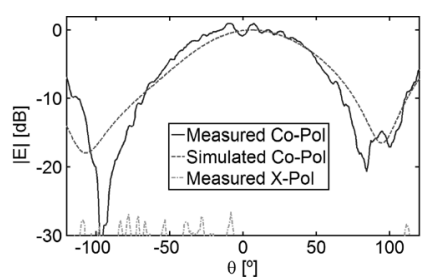

a)

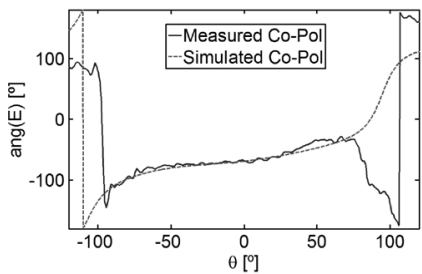

c)

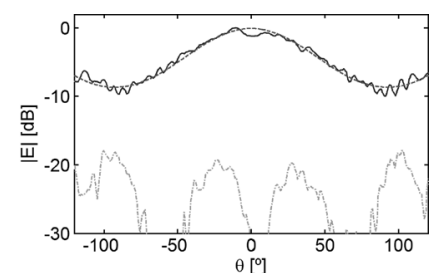

b)

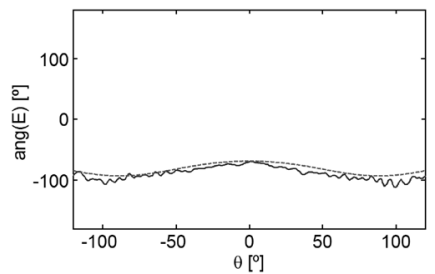

d)
Fig. 6. Measured and simulated radiation patterns at $f=7 \mathrm{GHz}$ : a) E-plane magnitude; b) H-plane magnitude; c) E-plane phase; d) H-plane phase.

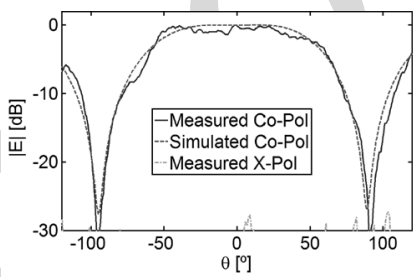

a)

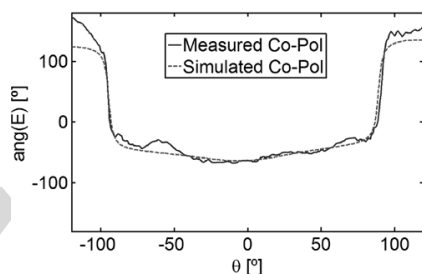

c)

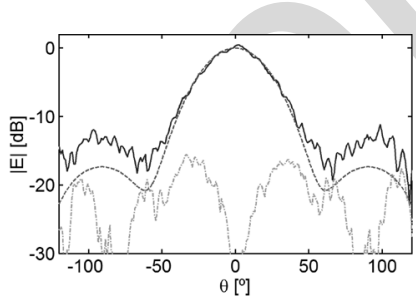

b)

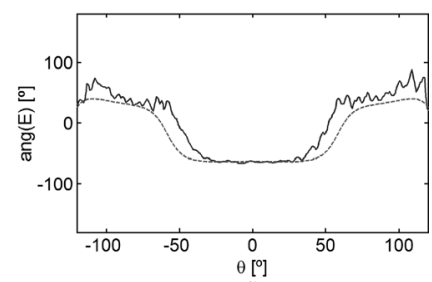

d)
Fig. 7. Measured and simulated radiation patterns at $f=9 \mathrm{GHz}$ : a) E-plane magnitude; b) H-plane magnitude; c) E-plane phase; d) H-plane phase.

CST simulated curve. A good impedance match is obtained over the entire UWB bandwidth from 3.1 to $10.6 \mathrm{GHz}$ (delimited by the two dashed vertical lines in Fig. 4) corresponding approximately to $110 \%$ bandwidth.

\section{A. Radiation Patterns}

Radiation patterns were measured for several frequencies across the band. Examples of magnitude and phase radiation pattern are shown in Figs. 5-7 for three different frequencies within the UWB band, superimposed on CST simulation curves. The $|\theta|>120^{\circ}$ region is omitted because it is blocked by the antenna tower, but the radiation pattern is bidirectional as previously referred. Part of the small ripple is originated by the reflection of the antenna back lobe on the antenna supporting tower. The excitation of the balanced antenna by the coaxial cable originates a slight lack of symmetry in the E-plane magnitude and phase radiation pattern as well as contribution to the ripple due to induced currents. Anyway the agreement with CST prediction is good, showing an almost 
constant phase characteristic which translates into a well defined constant phase centre across the elevation angle $\theta$. The measured cross-polarization is comfortably bellow $-25 \mathrm{~dB}$ in the E-plane and $-15 \mathrm{~dB}$ in the H-plane showing excellent polarization purity.

A slight increase in directivity with frequency is observed, mainly because of the narrowing of the radiation pattern in the $\mathrm{H}$-plane. However, from $8 \mathrm{GHz}$ onwards, this effect is counteracted by the broadening of the E-plane radiation pattern. CST simulations indicate that the directivity is about $4 \mathrm{dBi}$ at the lower part of the UWB band, it increases to about $6 \mathrm{dBi}$ at 8 $\mathrm{GHz}$ and then drops to about $3 \mathrm{dBi}$ at the upper end of the UWB band. These directivity results are confronted ahead with measurements.

The total antenna efficiency versus frequency, predicted by the CST model ranges from $90 \%$ to $97 \%$ across the bandwidth. This was confirmed by measurement at the lower frequencies.

\section{B. Transfer Functions}

Two equal XETS antennas are used for simulation and experimental link transfer function studies in this section. The antenna normalized vector transfer functions in transmit and receive modes $\vec{A}_{n, T x}(f, \theta, \phi)$ and $\vec{H}_{n, R x}(f, \theta, \phi)$, relate the radiated electric field to the driving antenna voltage and relate the received voltage to the incident electric field, respectively [2]. For the same antenna and following from time domain reciprocity, these functions relate as [31]

$$
A_{n, T x}=\frac{j \omega}{2 \pi c} H_{n, R x} .
$$

For equal parallel antennas, the ratio $s_{21}$ between the output voltage at the receiving antenna and the applied voltage at the transmitting antenna can be written as [13]

$$
s_{21}(f, \theta, \phi)=\frac{j \lambda}{4 \pi R}\left[H_{n, R x}^{\prime}(f, \theta, \phi)\right]^{2} \mathrm{e}^{-j 2 \pi f \frac{R}{c}}
$$

where $R$ is the distance between both antennas, and $H_{n, R x}^{\prime}=$ $H_{n, R x} \sqrt{4 \pi} / \lambda$.

Measurements of $s_{21}$ were performed in anechoic chamber using a Vector Network Analyzer (Agilent PNA E8361A) and two identical face-to-face co-polarized XETS antennas separated by $R=50 \mathrm{~cm}$. Prior full-port calibration referred to the antennas input ports was performed in order to remove frequency dependence from the RF cables. In this set-up the supporting structure was made from low-density polyurethane foam to minimize structure influence on the measurements. Fig. 8 shows the measured $S_{21}$ and the corresponding CST simulated curve, which agree quite well.

The magnitude of the transfer function is reasonably constant up to $8.5 \mathrm{GHz}$ and the unwrapped phase is almost linear over the entire UWB band which is expected to translate into low distortion of a transmitted UWB pulse. a)

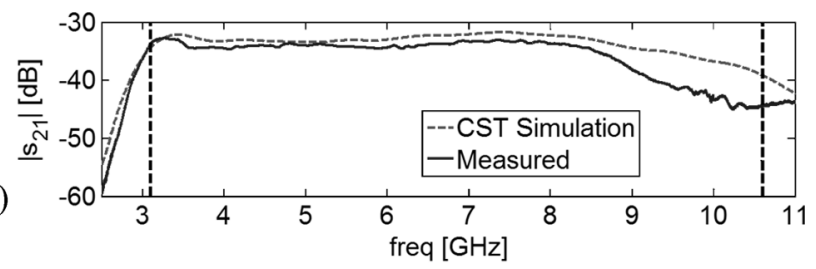

b)

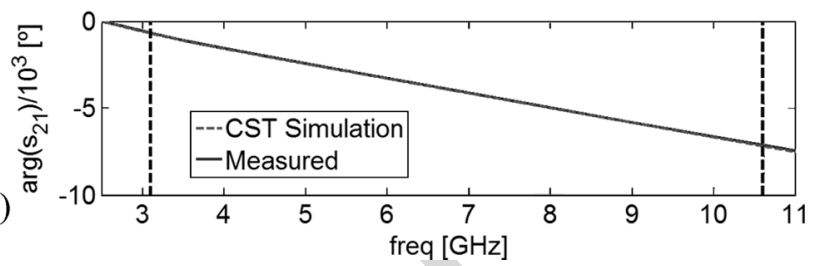

Fig. 8. Link transfer function between two identical face-to-face XETS antennas separated by $R=50 \mathrm{~cm}$ : a) amplitude; b) phase.

a)

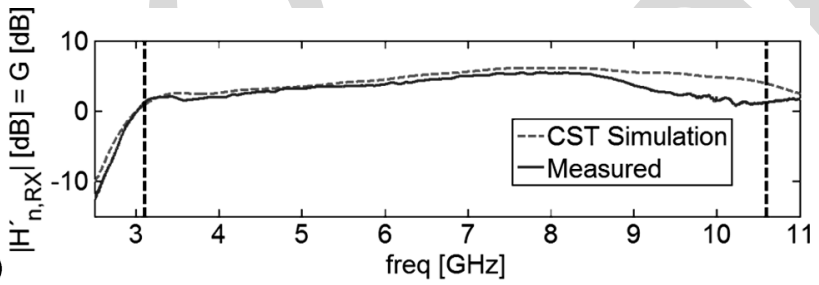

b)

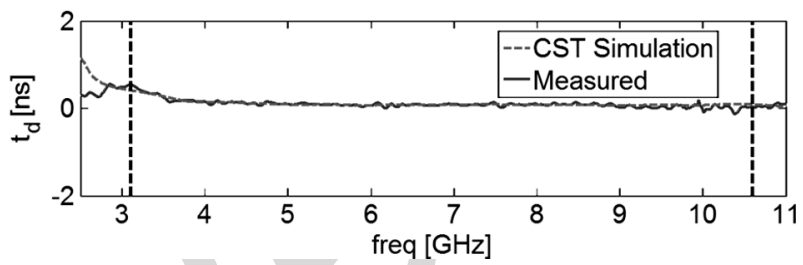

Fig. 9. a) Magnitude of $H_{n, R x}^{\prime}(\theta=\phi=0)$, which is coincident with the CW effective antenna gain in $\mathrm{dB}$ scale; b) group delay of the XETS.

Function $H_{n, R x}^{\prime}$ directly relates to continuous wave $\mathrm{CW}$ effective gain of the antenna [13], [31]

$$
\left|H_{n, R x}^{\prime}(f, \theta, \phi)\right|=\sqrt{G(f, \theta, \phi)} .
$$

Function $H_{n, R x}^{\prime}$ can be readily computed from (3) as a square root, and the result is shown in Fig. 9(a). It confirms the previously referred gain dependence versus frequency that was observed in the radiation patterns of Fig. 5 to Fig. 7.

Measured group delay is shown in Fig. 9(b), superimposed on CST simulated curve. The equivalent electrical length of the short feeding cable that is soldered to the antenna has been subtracted. Group delay is not only quite constant over the whole band but it is further very close to zero $(<500 \mathrm{ps})$. This means that the phase centre of the antenna remains very close to the antenna plane over the entire UWB bandwidth.

Additional measurements were performed for other $(\theta, \phi)$ antenna orientations with one XETS antenna fixed while rotating the other XETS antenna in its E- and H-plane as would be done for radiation pattern measurement. Results showed similar type 


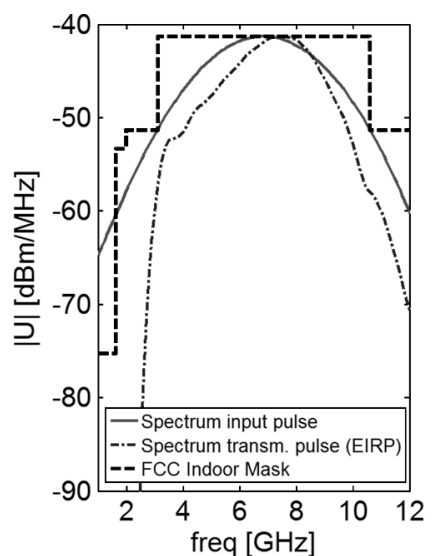

a)

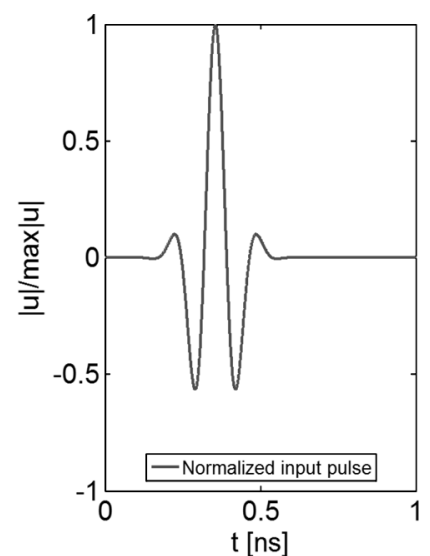

b)
Fig. 10. a) FCC indoor mask and complying spectrum of a test Gaussian pulse; b) corresponding pulse in time domain.

of frequency response and agreement with simulations. Both are presented ahead, in terms of time domain parameters.

\section{TIME DOMAIN PERFORMANCE}

\section{A. Input Pulse Characterization}

The performance of a UWB antenna can be quantified by the degree of distortion that it introduces in the transmission of a pulse. This distortion not only depends upon the antenna transfer function but also on the input waveform and the associated spectrum [32]. The test pulse adopted in the present work is defined as

$$
u(t)=\cos \left(2 \pi f_{c} t\right) \exp \left[-2 \pi(t / \tau)^{2}\right]
$$

where the central frequency is $f_{\mathrm{c}}=6.85 \mathrm{GHz}$ in order to center the pulse spectrum within the UWB band and the Gaussian width is $\tau=228$ ps to comply with the FCC indoor spectrum mask shown in Fig. 10(a). This mask limits power emission of the UWB radios to avoid possible interference with other radio systems operating within the same band [1]. The pulse amplitude in time and frequency domains is presented in Fig. 10. The entire band from 3.1 to $10.6 \mathrm{GHz}$ is used in order to produce the shortest possible pulse. In this respect, the indoor mask is more demanding to test the antenna performance than the outdoor mask. Fig. 10 also shows the transmitted pulse spectrum (EIRP at $1 \mathrm{MHz}$ bandwidth of single XETS for $\theta=\phi=0^{\circ}$ ) which fully complies with the FCC indoor mask.

The pulse defined in (5) is a good approximation of the up-converted triangular pulse which was demonstrated in [33] using CMOS technology for low-complexity, low-cost, low power consumption UWB transceivers. Simulations (not presented here) show that both pulse functions yield very similar results. Function (5) is adopted for possible comparison of the XETS results with other antenna results existing in the literature for the same excitation.

\section{B. Two-XETS Link Performance}

The measured link transfer function $s_{21}$ presented in Fig. 8, is used to obtain the shape of the received pulse at the second

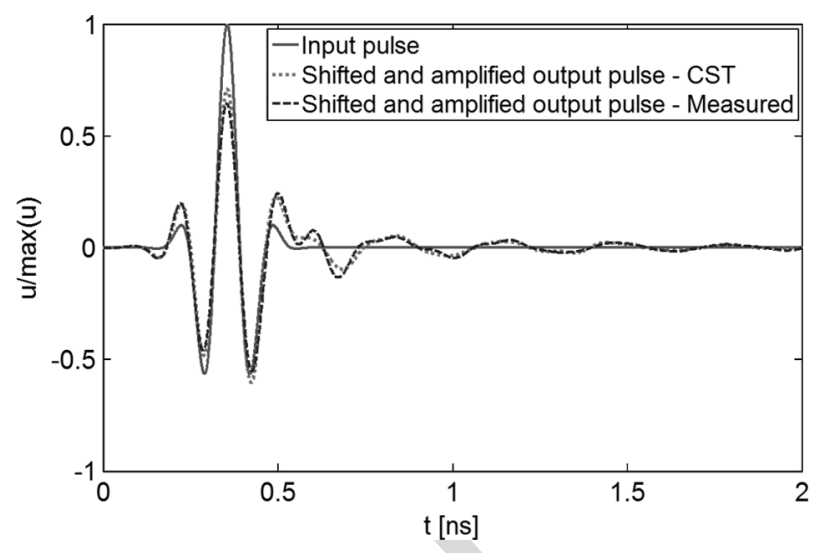

Fig. 11. Input and output pulse in time domain for a UWB link with two identical face-to-face XETS antennas.

antenna. It is represented by the dashed (blue) curve in Fig. 11 and CST simulation is superimposed (dotted magenta curve). Both curves agree quite well.

For better comparison between input and output pulses, the latter was normalized to the maximum value of $\left|s_{21}\right|$ and shifted in time. The output pulse shows good preservation of the input shape, although ringing inevitably appears at the trailing edge stretching the pulse duration. Two figures of merit can be used for more objective characterization of the output pulse distortion. One is the pulse fidelity $F(\theta, \phi)$ [2], and the other is based on the time window containing $90 \%$ of the pulse energy [3], [34], onwards referred as the $\operatorname{E} 90(\theta, \phi)$. The ratio of E90 time windows computed for the output and input pulses gives a measure of the time stretch of the output pulse introduced by the link.

For the pulse in Fig. 11, corresponding to the face-to-face two-antenna link, the measured result gives $F=93.1 \%, \mathrm{E} 90=$ $0.25 \mathrm{~ns}$, against $F=94.9 \%, \mathrm{E} 90=0.23 \mathrm{~ns}$ for the simulation prediction. For the input pulse E90 $=0.15 \mathrm{~ns}$, so the output pulse stretch ratio is 1.67 for measurements and 1.53 for simulation. The obtained values are compatible with envisaged UWB applications, allowing bit rates of hundreds of Mbit/s.

\section{Single Antenna Performance}

It would be interesting to investigate the antenna transient performance parameters over the whole solid angle, instead of just the axial direction. However this is not feasible with the used measurement setup except for the main planes. The same happens with the corresponding two-antenna CST simulation due to excessive required computational effort.

The alternative is to characterize a single antenna in the transmit mode. The distortion parameters definition now involve the detected E-field $E(t, \theta, \phi)$ pulse instead of the received voltage pulse in the receive antenna. It is clear that distortion parameter values obtained for a single XETS are different from those obtained for the two-antenna link, yet they are also meaningful and important.

In CST simulation this analysis can be done using a far-field linear polarized electrical field probe over the antenna solid angle [28]. In the experimental set-up, the spectrum of the transmitted E-field at an observation point at distance $R$ can be 


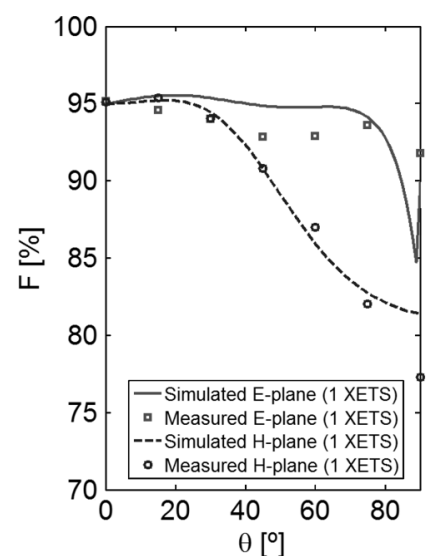

a)

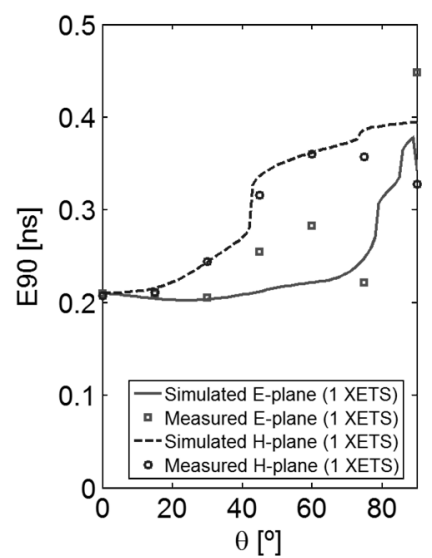

b)
Fig. 12. Output pulse distortion parameters for a single XETS antenna. a) Fidelity; b) E90 time window (the E90 window for the input pulse is $0.15 \mathrm{~ns}$ ).

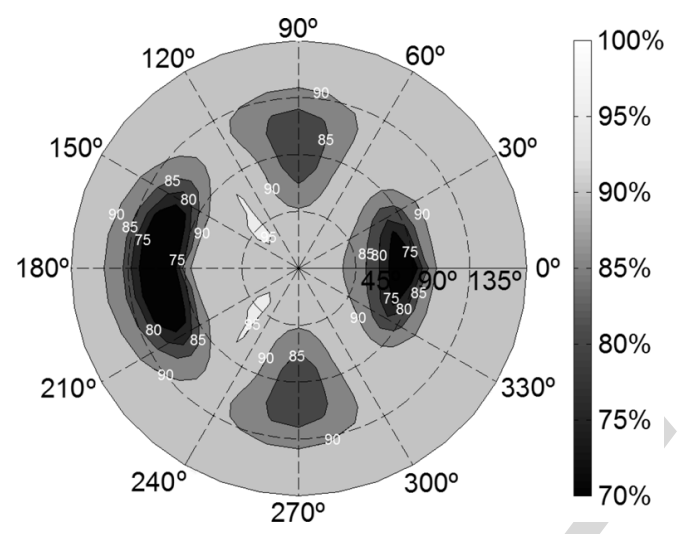

Fig. 13. Fidelity of a single XETS antenna over the soli angle. The radial angle is theta and the polar angle is phi.

obtained from the measured transfer function of the two-XETS link [2]

$$
\frac{E(f, R, \theta, \phi)}{\sqrt{120 \pi}}=\frac{j}{\sqrt{4 \pi} R} \frac{U(f)}{\sqrt{Z_{\text {in }}}} H_{n, R x}^{\prime}(f, \theta, \phi) \mathrm{e}^{-j 2 \pi f \frac{R}{c}}
$$

where $Z_{\text {in }}$ is the transmitting antenna characteristic port impedance and $U(f)$ is the spectrum of the applied voltage.

Measured and simulated values of the pulse distortion parameters are shown in Fig. 12 for the E- and H-planes of a single XETS antenna. Fidelity values range from $77.3 \%$ to $95.4 \%$ and E90 time window range from $0.21 \mathrm{~ns}$ to $0.45 \mathrm{~ns}$.

It is noted that the agreement between simulation and measurement is good, which allows exploring further the antenna properties using CST simulations. Fig. 13 presents the calculated fidelity of a single XETS antenna over the whole solid angle. The value ranges from $70 \%$ in the E- and $\mathrm{H}$-planes near $\theta=90^{\circ}$, to $95 \%$ in most of the front and back hemispheres. The $F$ drop close to $\theta=90^{\circ}$ is originated by the XETS radiation null in that direction; see Fig. 5(a) to Fig. 7(a). The $F$ drop is less abrupt in the H-plane close to $\theta=90^{\circ}$ because the radiation level is higher in this direction, see Fig. 5(b) to Fig. 7(b). The slight lack of overall symmetry is due to the feeding coaxial cable.

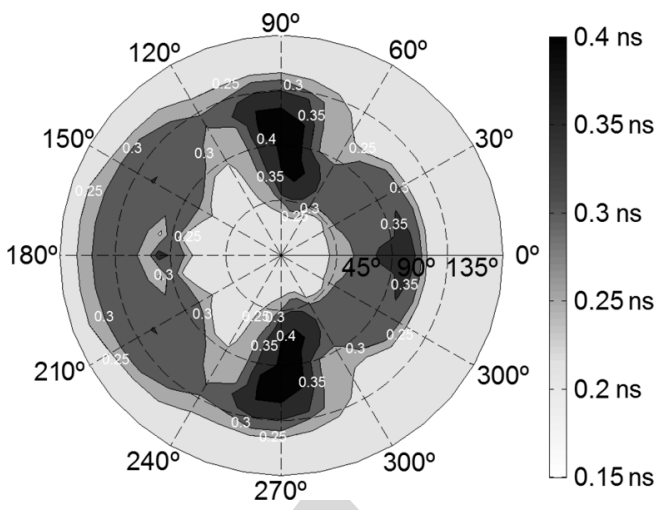

Fig. 14. Time window containing $90 \%$ of the pulse energy transmitted by a XETS antenna over the solid angle. The radial angle is theta and the polar angle is phi (the E90 window for the input pulse is $0.15 \mathrm{~ns}$ ).

The E90 time window results are presented in Fig. 14. The values range from $0.21 \mathrm{~ns}$ in the front and back directions to $0.41 \mathrm{~ns}$ in the $\mathrm{H}$-plane close to the $\theta=90^{\circ}$. However, while in the E-plane the larger pulse stretch is confined around $\theta=90^{\circ}$, the same does not happen in the H-plane. This is a consequence of the stronger frequency dependence of the H-plane radiation pattern. It is also relevant to point out that the maximum E90 time window of a single XETS antenna in the axial direction is only $0.21 \mathrm{~ns}$ while in the simulated complete radio link (Fig. 11) it is $0.23 \mathrm{~ns}$; in the latter case two antennas contribute for the pulse distortion.

So, safe for a little drop at the plane that contains the antenna, $F$ and E90 values are very good and stable over the solid angle, showing low pulse distortion.

\section{MultiAntenna ARrangements}

It was mentioned in the introduction that complementary steps for UWB radio development are related with the combination of this technology with the diversity and even with multiple-input-multiple-output (MIMO) concepts to enhance transmission data-rates and range [22], [23]. Spatial and polarization diversity can be explored in the above context; this requires good isolation between adjacent antennas, and between polarization diversity channels. This type of study on the UWB antenna part is just starting to appear in the literature [35].

The proposed XETS antenna presents excellent characteristics in this respect. The back petals shown in Fig. 1 for attaching the excitation to the XETS antenna can be repeated also for the orthogonal pair of petals to produce an almost independent orthogonal polarization mode in the same antenna. This configuration constitutes a solution of its own and it is not addressed here. Instead, this section quantifies the coupling between adjacent antennas in the E-plane, H-plane and mixed E- and H-planes (see Fig. 15). This coupling is evaluated trough the $s_{21}$ parameter across the UWB band and through the ratio $\varepsilon$ between the energy contained in the pulse received in one antenna to the energy transmitted in the adjacent antenna.

In this characterization the adjacent antennas are coplanar, printed on a common substrate and its centers are separated by the minimum distance $D_{\text {front }}$, that is $35 \mathrm{~mm}\left(0.3 \lambda_{0}\right)$. The results are summarized in Fig. 16. It is clear that the crossed 

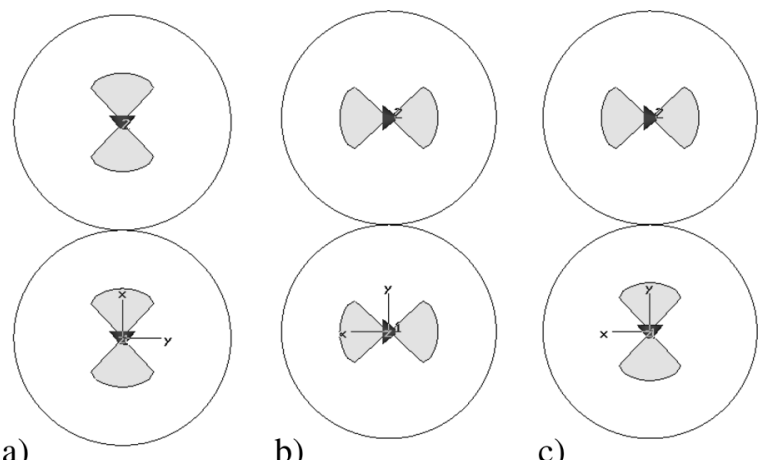

c)

Fig. 15. Double-antenna arrangement: a) E-plane; b) H-plane; c) crossed arrangement.

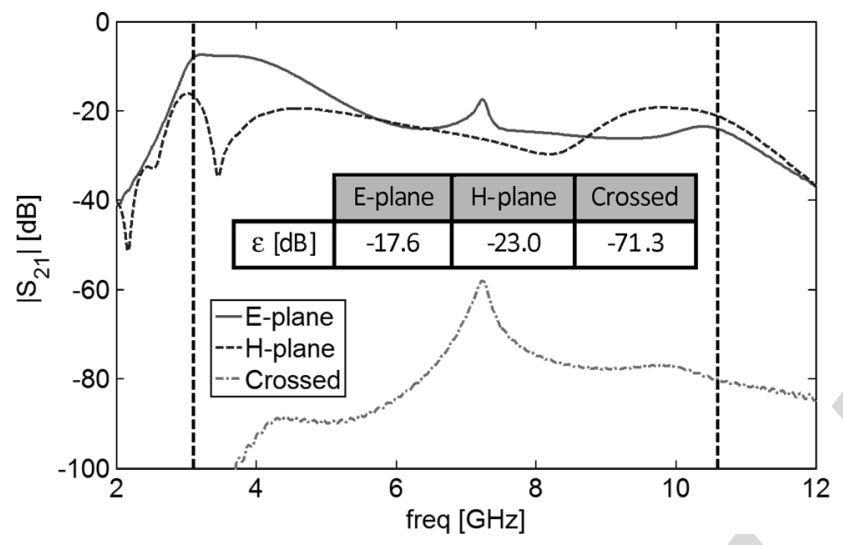

Fig. 16. Coupling between two XETS antennas in the different double arrangements. The inset table presents the ratio between the energy in the received pulse in one antenna to the energy transmitted by the adjacent antenna.

arrangement yields the best isolation, but even the H-plane arrangement is quite satisfactory. It should be mentioned that the good H-plane isolation for a similar antenna in a different context (mm-wave lens feed array) has been confirmed experimentally [30].

Time domain simulations have further shown that, any of the tested double-antenna arrangements in Fig. 15 produces little change in the $F$ and E90 time window compared to the isolated antenna case (calculations based on transmit mode transfer function obtained with field probe).

\section{CONCLUSION}

The presented study confirmed with measurements shows that the XETS is a very good antenna candidate for UWB communications using the FCC assigned 3.1-10.6 GHz. The antenna diameter is $35 \mathrm{~mm}\left(0.3 \lambda_{0}\right.$ at $\left.3.1 \mathrm{GHz}\right)$ and substrate is 10 mil thick; so the antenna is compact and low-profile. Owing to the crossed exponential slots and to the antenna perfect two-plane symmetry, the radiation pattern presents smooth dependence with frequency and its linear polarization is shown to be completely stable for all observation angles, with low cross-polarization level and low mutual coupling in multi-antenna arrangements. This characteristic is not so common in other reported antenna solutions. It makes the XETS especially attractive for combining the UWB concept with diversity and
MIMO strategies to enhance link capacity and improve range, which is constrained by the FCC power emission mask for UWB. The balanced antenna configuration is adequate for direct integration of differential UWB RFICs at the back of the antenna, avoiding the need for an additional balun, thus decreasing the complexity of the UWB transceiver.

Time domain characterization of the XETS showed as well low pulse distortion effects, thoroughly quantified through the pulse fidelity parameter (ranging from $70 \%$ to $95 \%$ across the solid angle) and by the width of the $90 \%$ energy window (ranging from $0.21 \mathrm{~ns}$ to $0.41 \mathrm{~ns}$ ). The worse results correspond to the plane containing the printed antenna, which is unavoidable. These results are compatible with the envisaged several hundred Mbit/s data rates. Improved results would be obtained with a balanced feed.

It is interesting to mention that the proposed antenna configuration still leaves room for further developments. The addition of appropriately designed slots in the XETS can produce a notch in the antenna transfer function to prevent interference with WLAN bands and avoid extra dedicated filtering components in the system. These results will appear elsewhere. Other line for further developments is the duplication of the back-face petals to enable dual polarization excitation of the same XETS for achieving a very compact polarization diversity arrangement.

\section{ACKNOWLEDGMENT}

The authors acknowledge the collaboration from V. Fred and C. Brito for prototype construction, and A. Almeida for prototype measurements.

\section{REFERENCES}

[1] Federal Communications Commission (FCC), First Order and Report: Revision of Part 15 of the Commission's Rules Regarding UWB Transmission Systems FCC 02-48, Apr. 22, 2002.

[2] D. Lamensdorf and L. Susman, "Baseband-pulse-antenna techniques," IEEE Antennas Propag. Mag., vol. 36, pp. 20-30, Feb. 1994.

[3] M. Klemm and G. Tröster, "Characterization of small planar antennas for UWB mobile terminals," Wireless Commun. Mobile Comput., vol. 5, no. 5, pp. 525-536, Aug. 2005.

[4] Z. Chen et al., "Planar antennas," IEEE Microw. Mag., vol. 7, no. 6, pp. 63-73, Dec. 2006.

[5] T. Karacolak and E. Topsakal, "A double-sided rounded bow-tie antenna (DSRBA) for UWB communication," IEEE Antennas Wireless Propag. Lett., vol. 5, pp. 446-449, 2006.

[6] X. Wu, Z. Chen, and N. Yang, "Optimization of planar diamond antenna for single-band and multiband UWB wireless communications,' Microw. Opt. Technol. Lett., vol. 42, no. 6, pp. 451-455, Sept. 2004.

[7] J. Zhang, Y. Xu, and W. Wang, "Ultra-wideband microstrip-fed planar elliptical dipole antenna," Electron. Lett., vol. 42, no. 3, pp. 144-145, Feb. 2006.

[8] E. Gueguen, F. Thudor, and P. Chambelin, "A low cost UWB printed dipole antenna with high performance," in Proc. IEEE Inter. Conf. on Ultra-Wideband-ICU 2005, Zurich, Switzerland, Sep. 2005, pp. 89-92.

[9] S. Zhong, X. Liang, and W. Wang, "Compact elliptical monopole antenna with impedance bandwidth in excess of 21:1," IEEE Trans. Antennas Propag., vol. 55, no. 11, pp. 3080-3085, Nov. 2007.

[10] J. Liang et al., "Study of a printed circular disc monopole antenna for UWB systems," IEEE Trans. Antennas Propag., vol. 53, no. 11, pp. 3500-3504, Nov. 2005.

[11] A. Mohamed and L. Shafai, "Investigation on the phase centre of ultra wideband circular monopole antennas," presented at the IEEE Antennas Propag. Society Inter. Symp., San Diego, CA, Jul. 2008.

[12] W. Lui, C. Cheng, and H. Zhu, "Improved frequency notched ultrawideband slot antenna using square ring resonator," IEEE Trans. Antennas Propag., vol. 55, no. 9, pp. 2445-2450, Sep. 2007. 
[13] T. Ma and S. Jeng, "Planar miniature tapered-slot-fed annular slot antennas for ultrawideband radios," IEEE Trans. Antennas Propag., vol. 53, no. 3, pp. 1194-1202, Mar. 2005.

[14] I. J. Yoon et al., "Ultrawideband tapered slot antenna with band cutoff characteristic," Electron. Lett., vol. 41, no. 11, pp. 629-630, May 2005.

[15] C. Chen, W. McKinzie, and N. Alexopoulos, "Stripline-fed arbitrarily shaped printed-aperture antennas," IEEE Trans. Antennas Propag., vol. 45, no. 7, pp. 1186-1198, Jul. 1997.

[16] J. Martínez-Fernández, J. Gil, and J. Zapata, "Ultrawideband optimized profile monopole antenna by means of simulated annealing algorithm and the finite element method," IEEE Trans. Antennas Propag., vol. 55, no. 6, pp. 1826-1832, Jun. 2007.

[17] M. Ammann, R. Cordoba, M. Uzelac, J. Evans, and A. Schwarzbacher, "On pattern stability of the crossed planar monopole," Microw. Opt. Technol. Lett., vol. 40, no. 4, pp. 294-296, Feb. 2004.

[18] X. Li, S. Hagness, M. Choi, and D. van der Weide, "Numerical and experimental investigation of an ultrawideband ridged pyramidal horn antenna with curved launching plane for pulse radiation," IEEE Antennas Wireless Propag. Lett., vol. 2, pp. 259-262, 2003.

[19] J. Andrews, UWB Signal Sources, Antennas and Propagation Picosecond Pulse Labs, Application Note AN-30414a, pp. 1-11, Aug. 2003.

[20] M. Blech and T. Eibert, "A dipole excited ultrawideband dielectric rod antenna with reflector," IEEE Trans. Antennas Propag., vol. 55, no. 7 , pp. 1948-1954, Jul. 2007.

[21] S. Bruni, A. Neto, and F. Marliani, "The ultrawideband leaky lens antenna," IEEE Trans. Antennas Propag., vol. 55, no. 10, pp. 2642-2653, Oct. 2007.

[22] W. Malik and D. Edwards, "Measured MIMO capacity and diversity gain with spatial and polar arrays in ultrawideband channels," IEEE Trans. Commun., vol. 55, no. 12, pp. 2361-2370, Dec. 2007.

[23] J. Keignart, C. Abou-Rjeily, C. Delaveaud, and N. Daniele, "UWB SIMO channel measurements and simulations," IEEE Trans. Microw. Theory Tech., vol. 54, no. 4, pp. 1812-1819, Jun. 2006.

[24] A. Vorobyov et al., "Integration of a pulse generator on chip into a compact ultrawideband antenna," IEEE Trans. Antennas Propag., vol. 56, no. 3, pp. 858-868, Mar. 2008.

[25] Agilent-Ultra-Wideband Communication RF Measurements Application Note 1488, 2005.

[26] P. Datta, X. Fan, and G. Fischer, "A transceiver front-end for ultrawide-band applications," IEEE Trans. Circuits Systems-II: Express Briefs, vol. 54, no. 4, pp. 362-366, Apr. 2007.

[27] J. Costa and C. Fernandes, "Crossed exponentially tapered slot antenna for UWB applications," presented at the IEEE Antennas Propag. Society Inter. Symp., San Diego, CA, Jul. 2008.

[28] CST [Online]. Available: www.cst.com

[29] J. Costa and C. Fernandes, "Broadband slot feed for integrated lens antennas," IEEE Antennas Wireless Propag. Lett., vol. 6, pp. 396-400, 2007.

[30] J. Costa and C. Fernandes, "Integrated imaging lens antenna with broadband feeds," presented at the 2nd Eur. Conf. on Antennas Propag.—EUCAP, Edinburgh, U.K., Nov. 2007.

[31] C. Baum, E. Farr, and C. Frost, "Transient gain of antennas related to the traditional continuous-wave (CW) definition of gain," in Proc. Ultra-Wideband Short-Pulse Electromagn., Jun. 1998, vol. 4, pp. 109-118.

[32] D. Pozar, "Waveform optimizations for ultrawideband radio systems," IEEE Trans. Antennas Propag., vol. 51, no. 9, pp. 2335-2345, Sep. 2003.

[33] J. Ryckaert, "Ultrawideband transmitter for low-power wireless body area networks: Design and evaluation," IEEE Trans. Circuits Syst., vol. 52 , no. 12 , pp. 2515-2525, Dec. 2005.
[34] N. Fortino, J.-Y. Dauvignac, G. Kossiavas, and R. Staraj, "Design optimization of UWB printed antenna for omnidirectional pulse radiation," IEEE Trans. Antennas Propag., vol. 56, no. 7, pp. 1875-1881, Jul. 2008.

[35] Z. Chen, "UWB antennas with enhanced performances," in Proc. Int. Conf. on Microw. and Millimeter Wave Technology, ICMMT 2008, Nanjing, China, Apr. 2008, vol. 1, pp. 387-390.

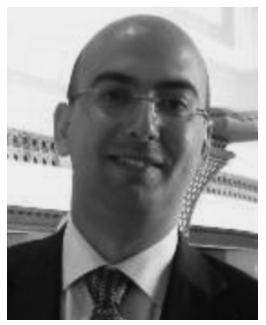

Jorge R. Costa (S'97-M'03) was born in Lisbon, Portugal, in 1974. He received the Licenciado and $\mathrm{Ph} . \mathrm{D}$. degrees in electrical and computer engineering from the Instituto Superior Técnico (IST), Technical University of Lisbon, Lisbon, Portugal, in 1997 and 2002 , respectively.

$\mathrm{He}$ is currently a Researcher at the Instituto de Telecomunicações, Lisbon, Portugal. He is also an Assistant Professor at the Departamento de Ciências e Tecnologias da Informação, Instituto Superior de Ciências do Trabalho e da Empresa. His present research interests include lenses, reconfigurable antennas, MEMS switches, UWB and RFID antennas.

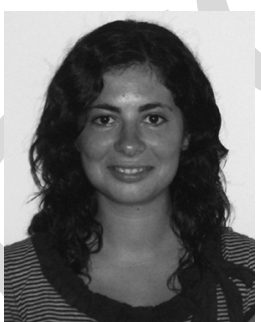

Carla R. Medeiros was born in Ponta Delgada, Açores, Portugal, in 1982. She received the Licenciado and M.Sc. degrees in electrical and computer engineering from the Instituto Superior Técnico (IST), Technical University of Lisbon, Lisbon, Portugal, in 2006 and 2007, respectively.

Since 2006, she has been a researcher at the Instituto de Telecomunicações (IT), focusing her work on antenna for wireless communications. She collaborates in national research projects. Her current research interests are in the areas of reconfigurable,

RFID and UWB antennas.

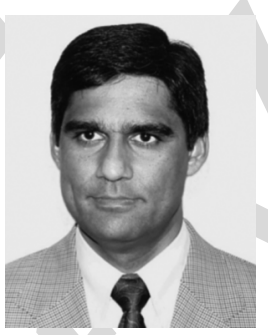

Carlos A. Fernandes (S'86-M'89-SM'08) received the Licenciado, M.Sc., and Ph.D. degrees in electrical and computer engineering from Instituto Superior Técnico (IST), Technical University of Lisbon, Lisbon, Portugal, in 1980, 1985, and 1990, respectively.

He joined the IST in 1980, where he is presently Full Professor at the Department of Electrical and Computer Engineering in the areas of microwaves, radio wave propagation and antennas. He is a Senior Researcher at the Instituto de Telecomunicações and Coordinator of its Wireless Communications scientific area. He has been the leader of antenna activities in National and European Projects as RACE 2067-MBS (Mobile Broadband System), ACTS AC230—SAMBA (System for Advanced Mobile Broadband Applications) and ESA/ESTEC-ILASH (Integrated Lens Antenna Shaping). He has coauthored a book, a book chapter, and more than 100 technical papers in international journals and conference proceedings, in the areas of antennas and radiowave propagation modeling. His current research interests include dielectric antennas for millimeter wave applications, antennas and propagation modeling for personal communication systems, RFID antennas, artificial dielectrics and metamaterials. 